\title{
New Phloroglucinol Derivatives from the Fruit Tree Syzygium jambos and Their Cytotoxic and Antioxidant Activities
}

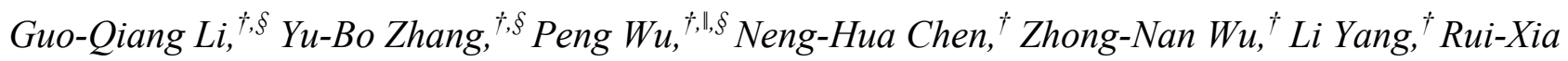
Qiu, ${ }^{\dagger}$ Guo-Cai Wang, ${ }^{* \dagger}$ Yao-Lan Li* ${ }^{\dagger}$

${ }^{\dagger}$ Institute of Traditional Chinese Medicine and Natural Products, College of Pharmacy, Jinan University, Guangzhou 510632, P. R. China

Department of Food Science and Engineering, Jinan University, Guangzhou 510632, P. R. China

"International Institute for Translational Chinese Medicine, Guangzhou University of Chinese Medicine, Guangzhou 510006, P. R. China 


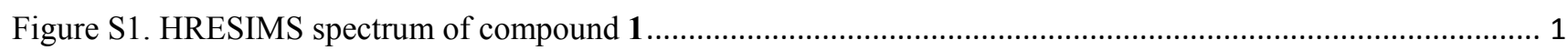

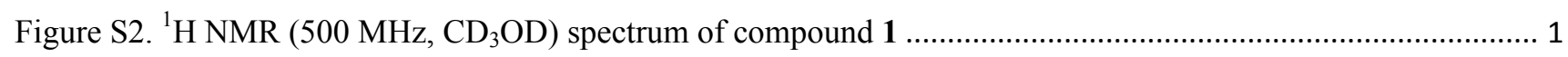

Figure $\mathrm{S} 3 .{ }^{13} \mathrm{C}$ NMR $\left(125 \mathrm{MHz}, \mathrm{CD}_{3} \mathrm{OD}\right)$ spectrum of compound 1 .............................................................. 2

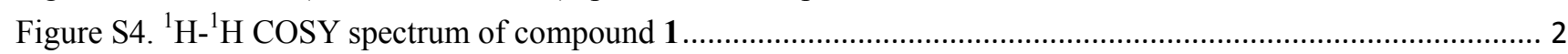

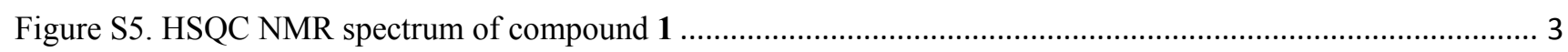

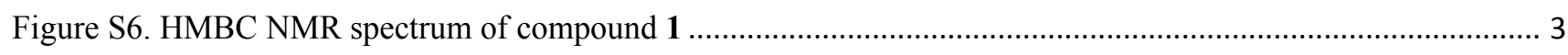

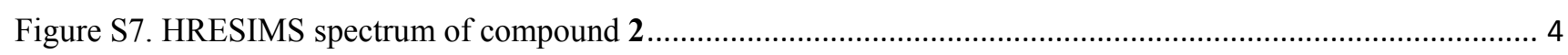

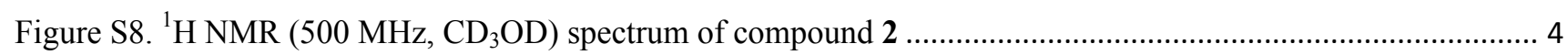

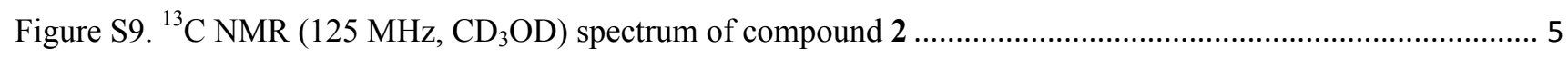

Figure S10. ${ }^{1} \mathrm{H}-{ }^{1} \mathrm{H}$ COSY spectrum of compound 2 …............................................................................... 5

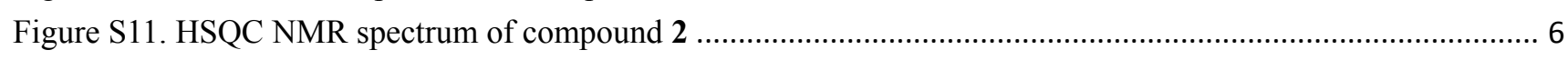

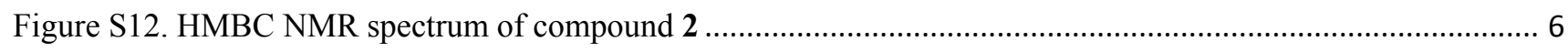

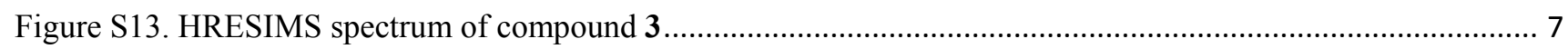

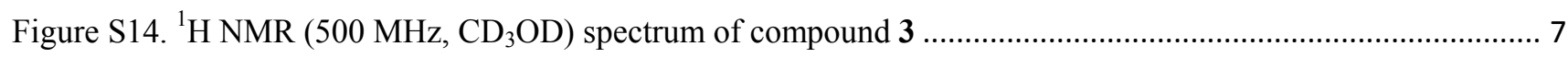

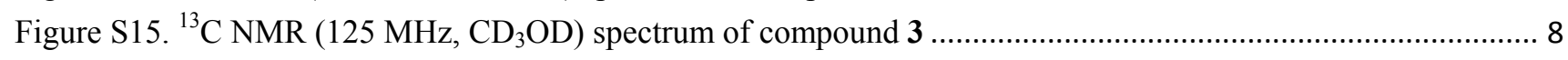

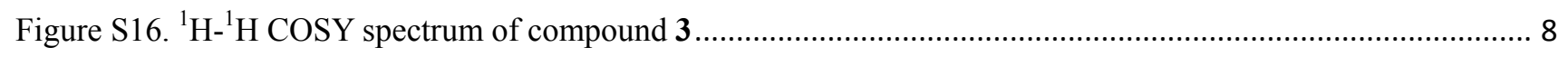

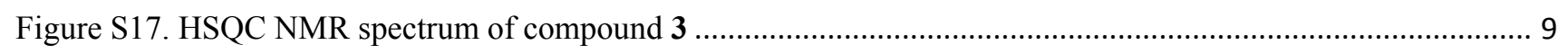

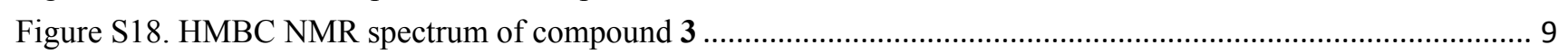

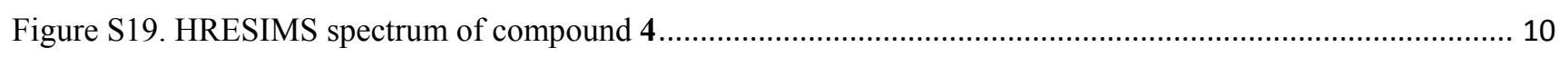

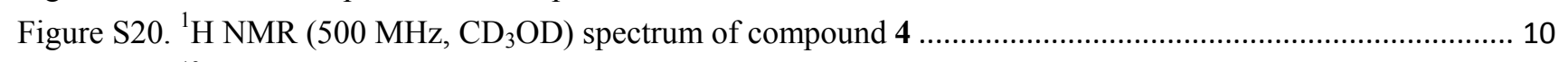

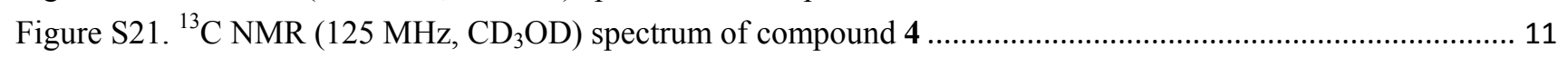

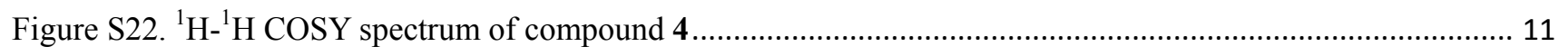

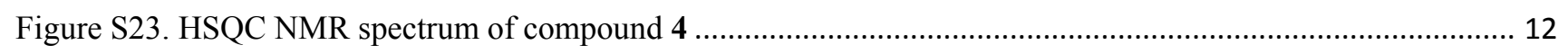

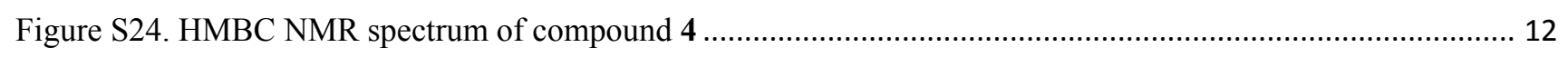

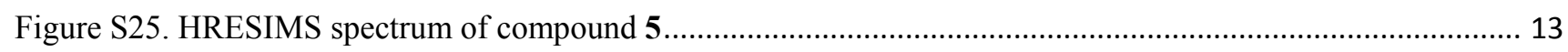

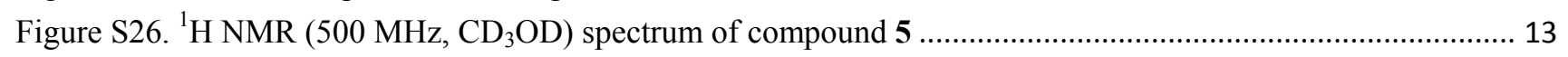

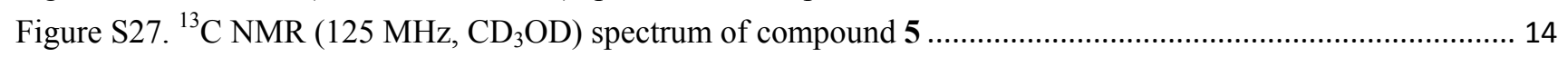

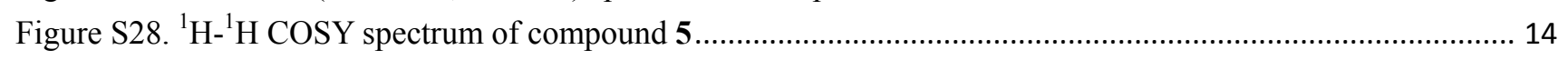

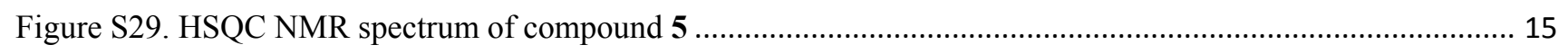

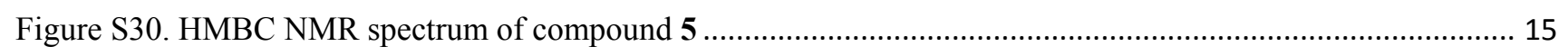

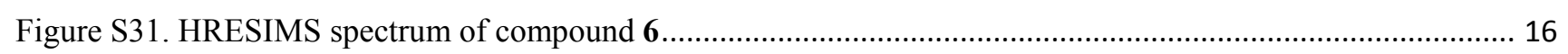

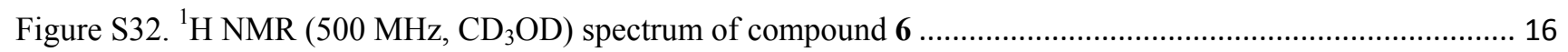

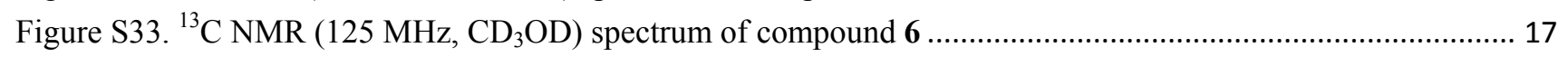

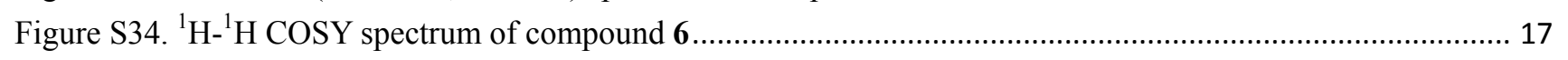

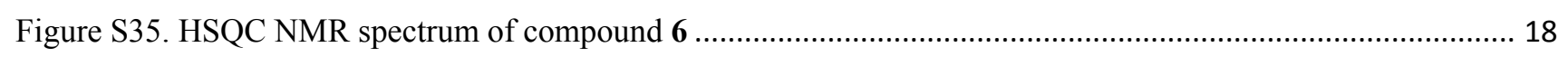

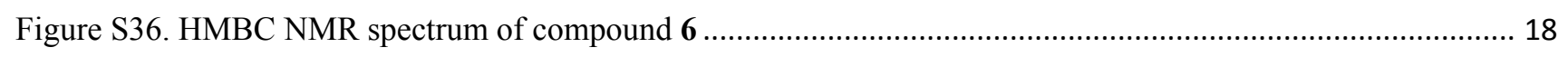

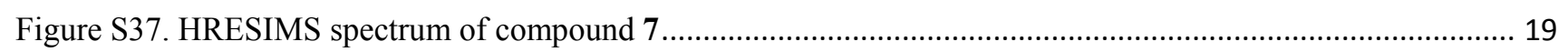

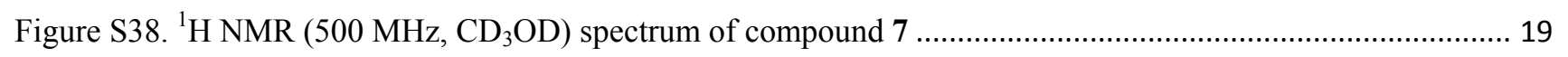

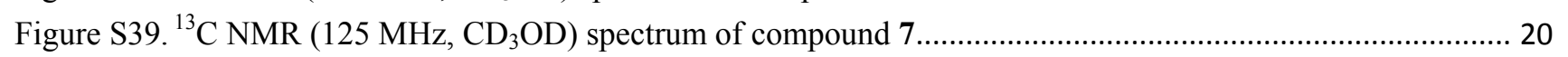

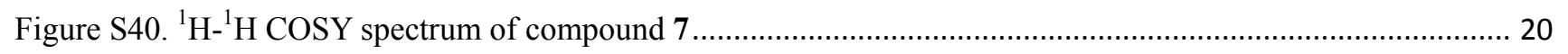

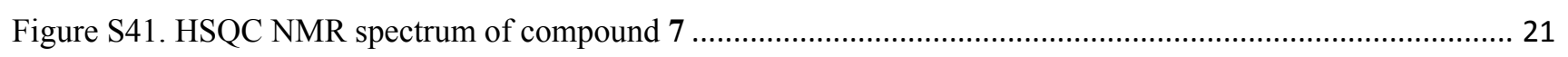

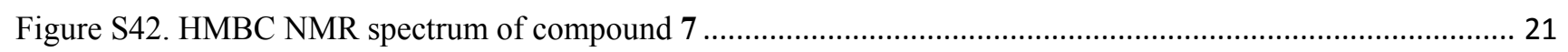




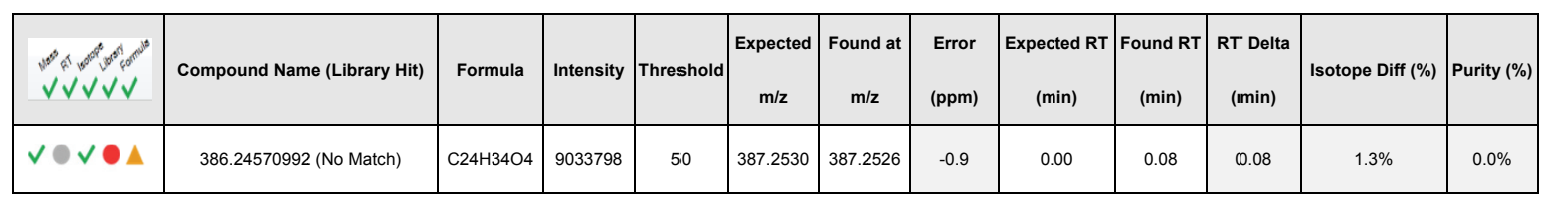

OSpectrum from Data5.wiff (sample 1) - Sample005, Experiment 1, +TOF MS (100 - 1000) from 0.144 to $0.168 \mathrm{~min}$ $\mathrm{OC} 24 \mathrm{H} 3404+\mathrm{H}$

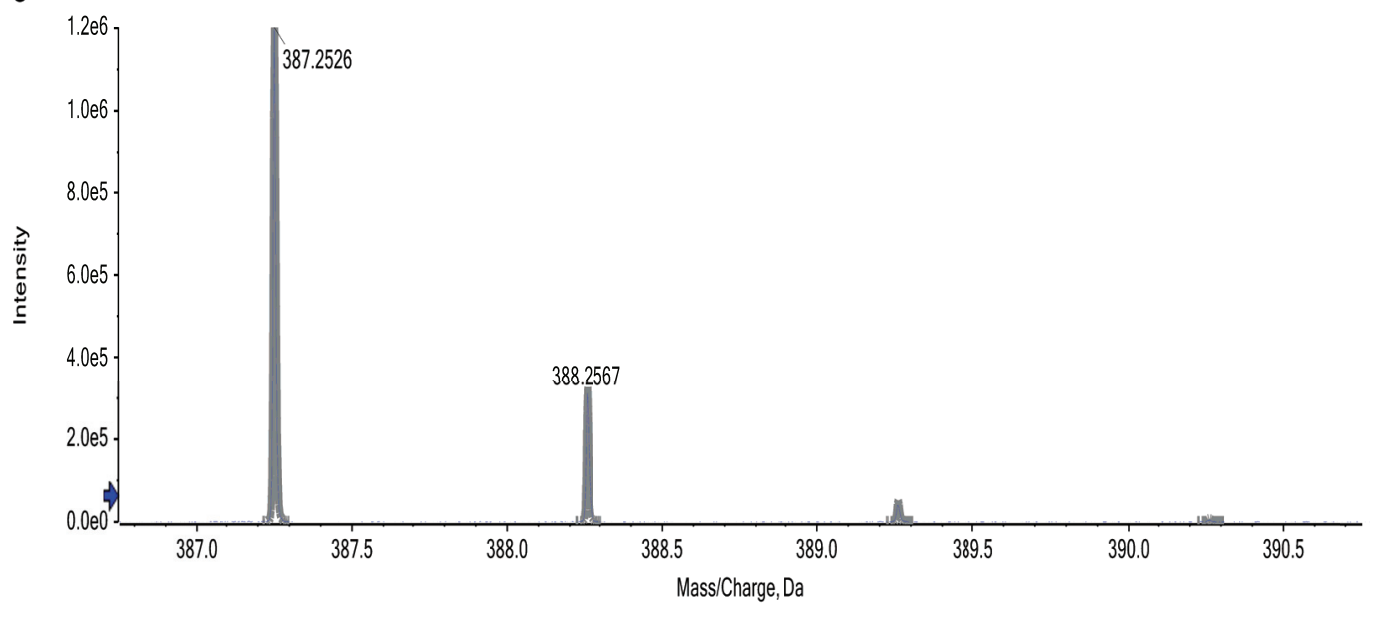

Figure S1. HRESIMS spectrum of compound 1

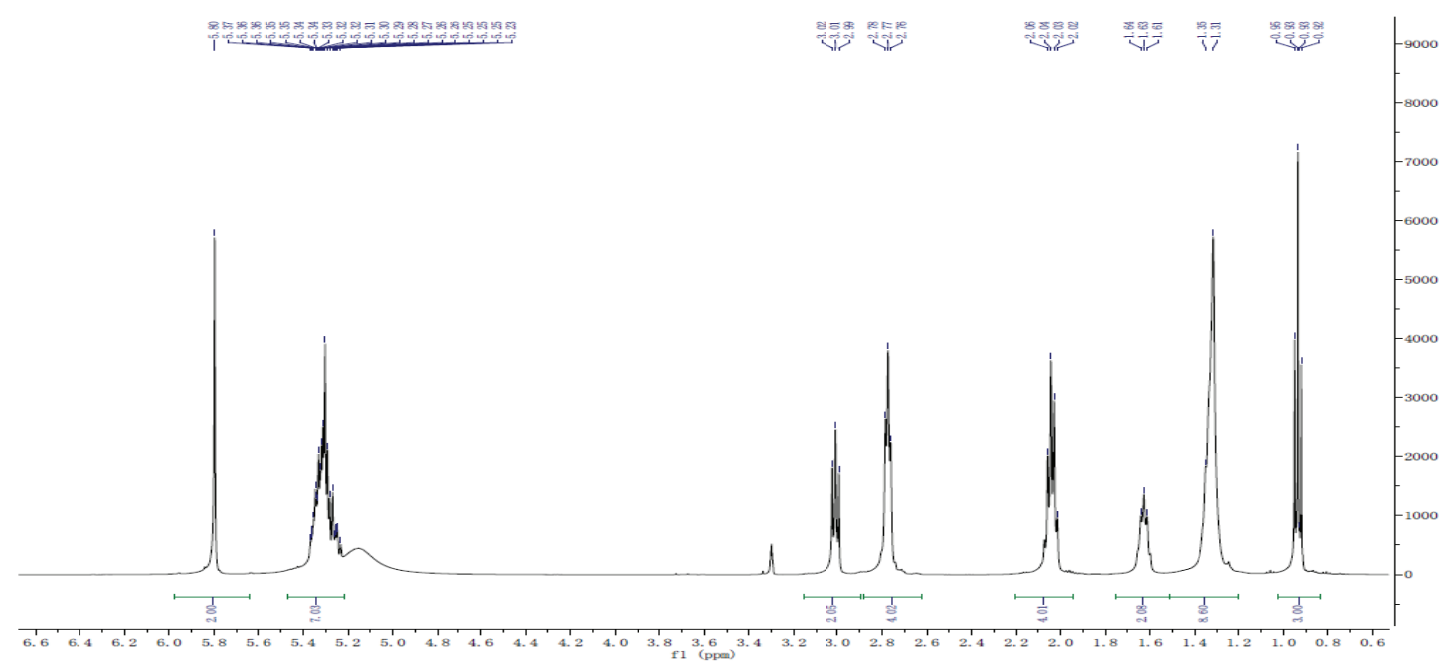

Figure S2. ${ }^{1} \mathrm{H}$ NMR (500 $\left.\mathrm{MHz}, \mathrm{CD}_{3} \mathrm{OD}\right)$ spectrum of compound 1 


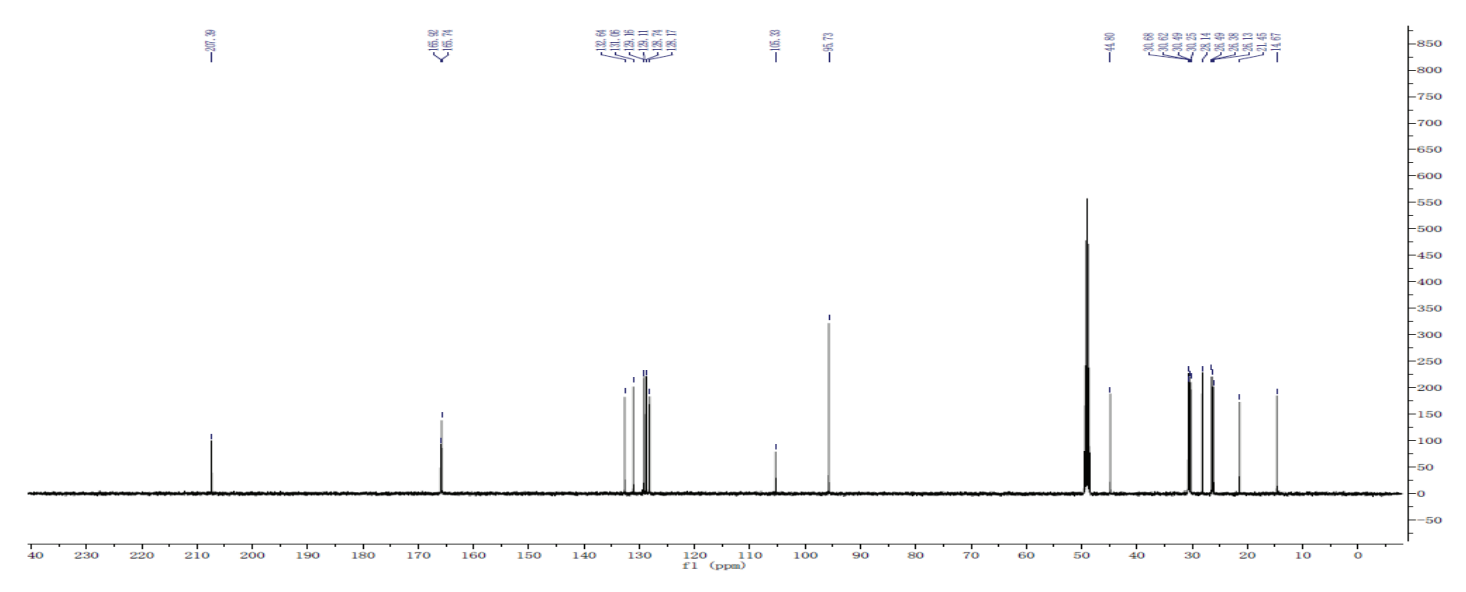

Figure S3. ${ }^{13} \mathrm{C}$ NMR (125 MHz, $\left.\mathrm{CD}_{3} \mathrm{OD}\right)$ spectrum of compound 1

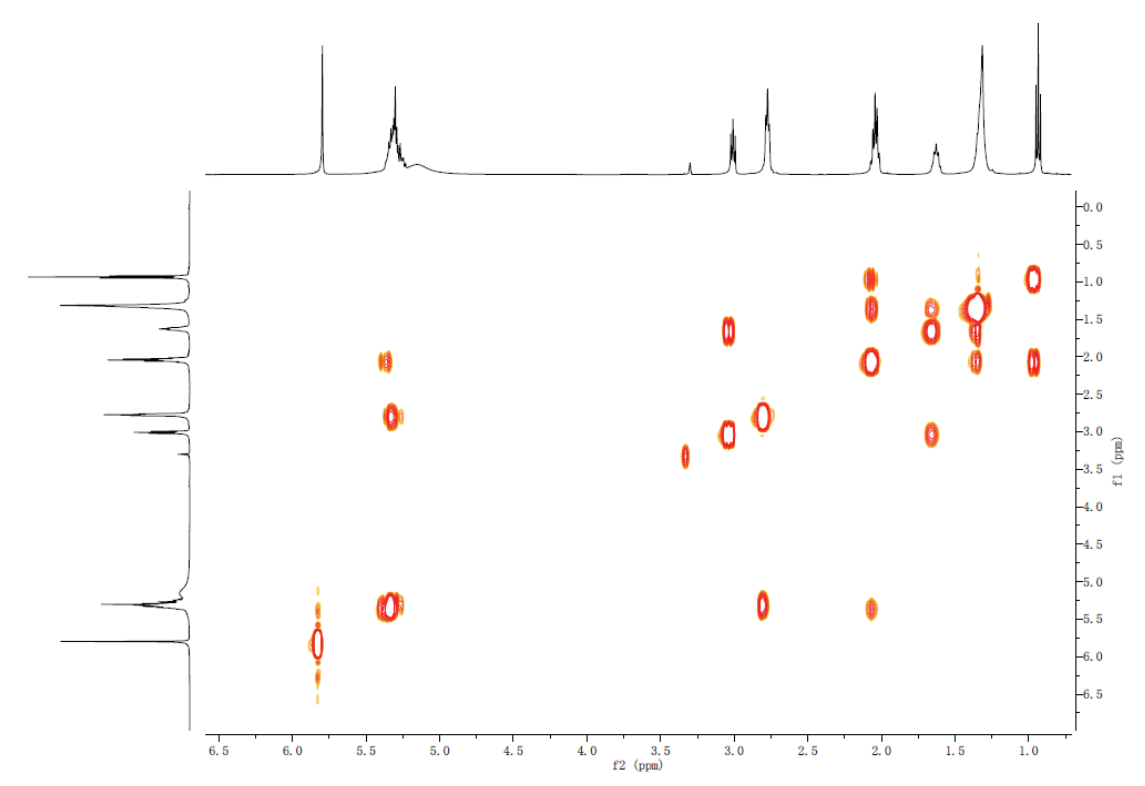

Figure S4. ${ }^{1} \mathrm{H}-{ }^{1} \mathrm{H}$ COSY spectrum of compound 1 


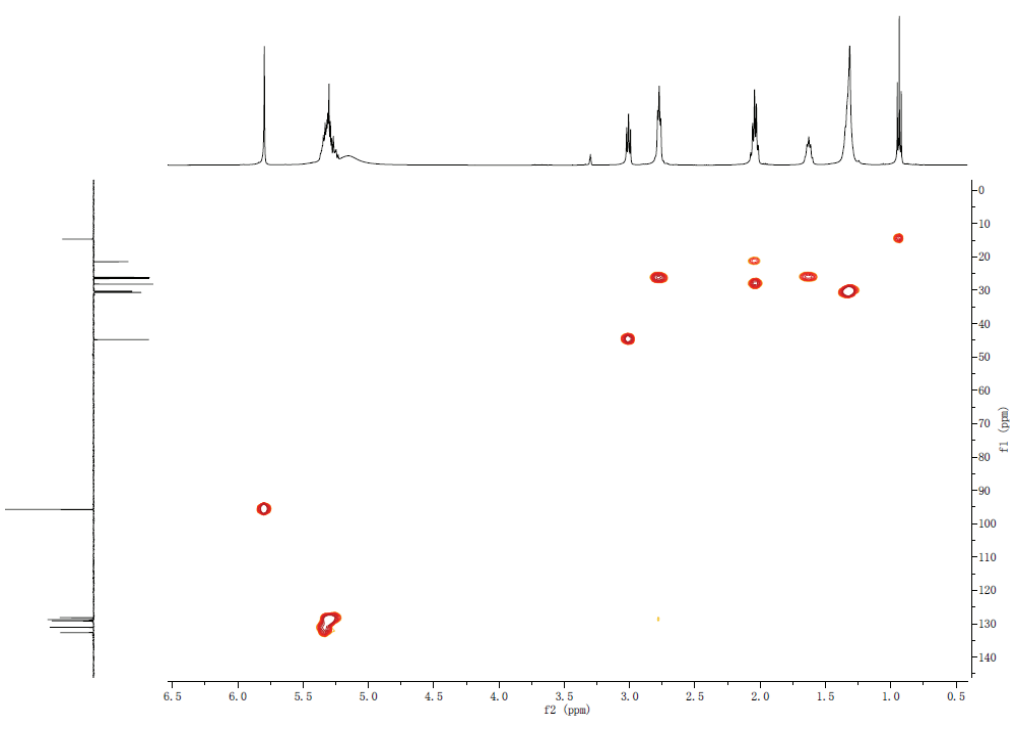

Figure S5. HSQC NMR spectrum of compound 1

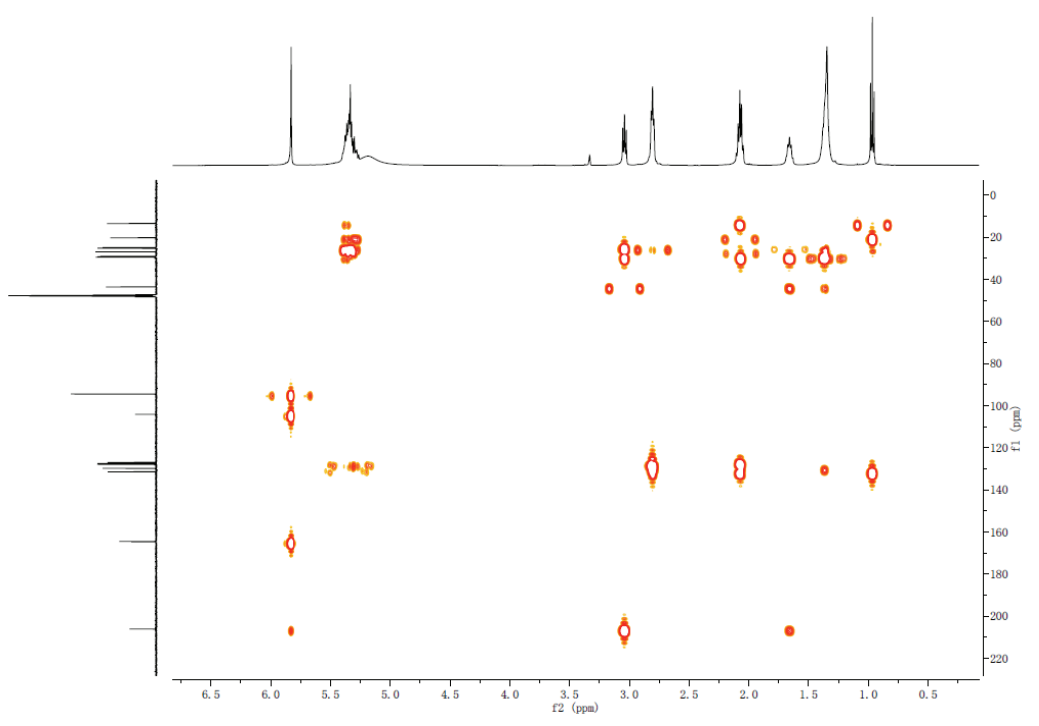

Figure S6. HMBC NMR spectrum of compound 1 


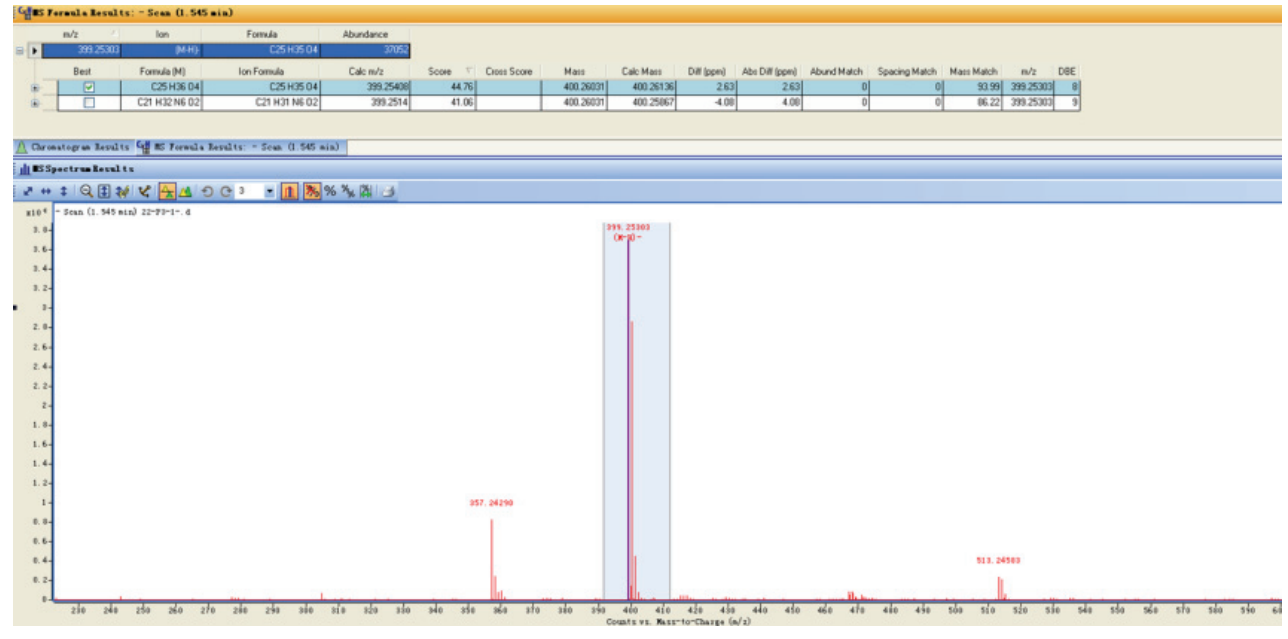

Figure S7. HRESIMS spectrum of compound 2

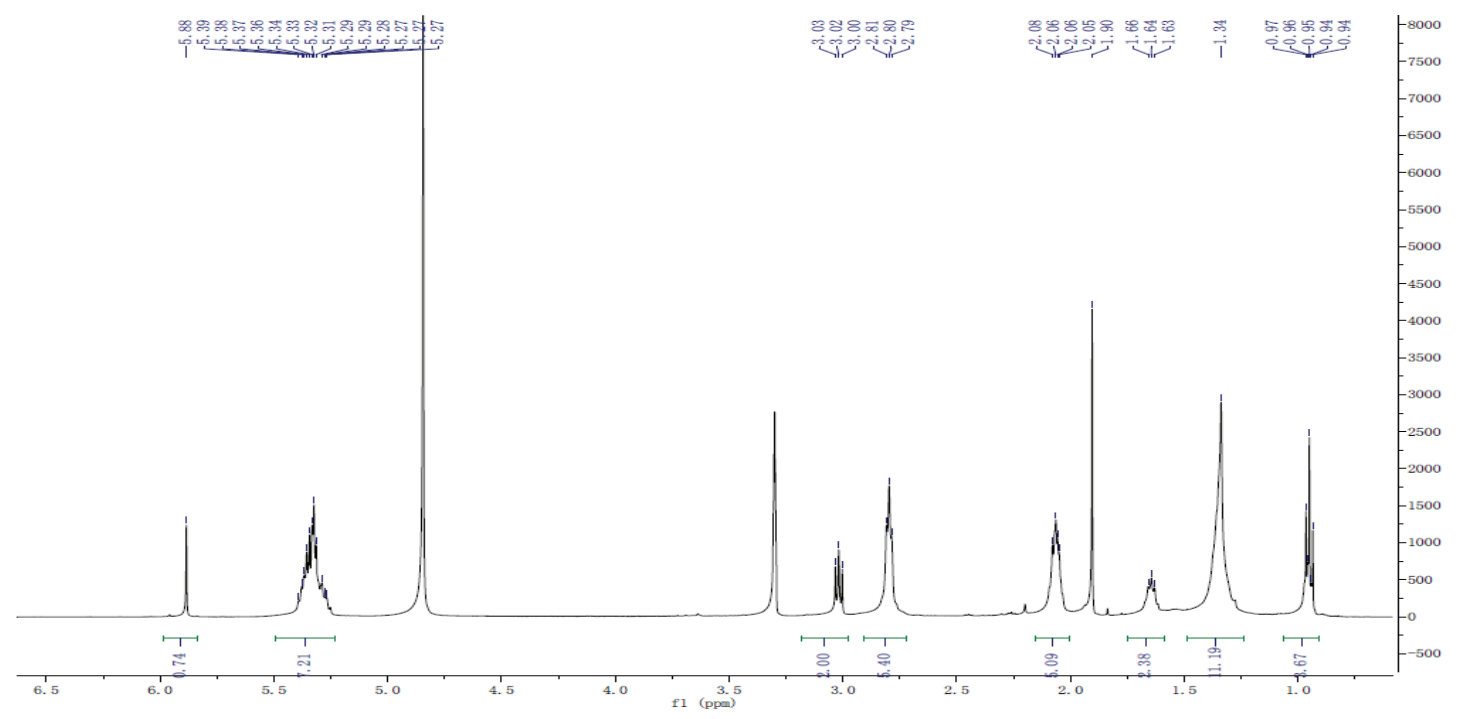

Figure S8. ${ }^{1} \mathrm{H}$ NMR (500 $\left.\mathrm{MHz}, \mathrm{CD}_{3} \mathrm{OD}\right)$ spectrum of compound 2 


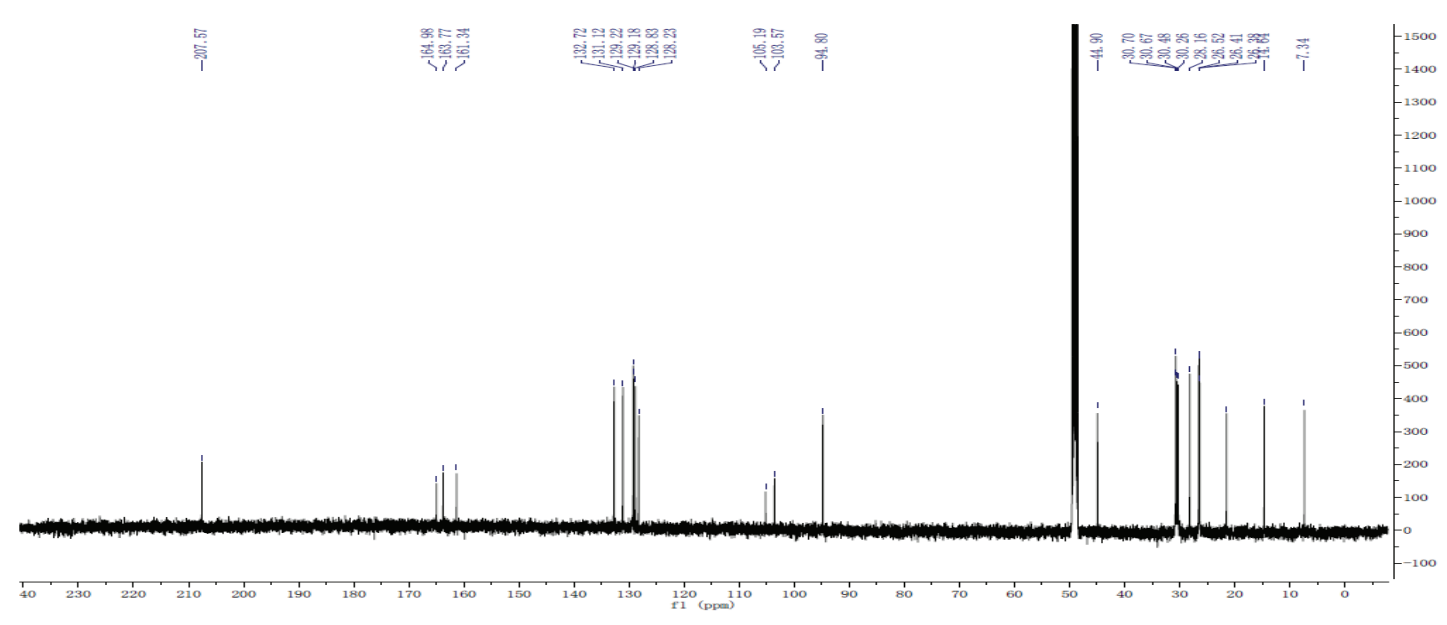

Figure S9. ${ }^{13} \mathrm{C}$ NMR (125 MHz, $\left.\mathrm{CD}_{3} \mathrm{OD}\right)$ spectrum of compound 2

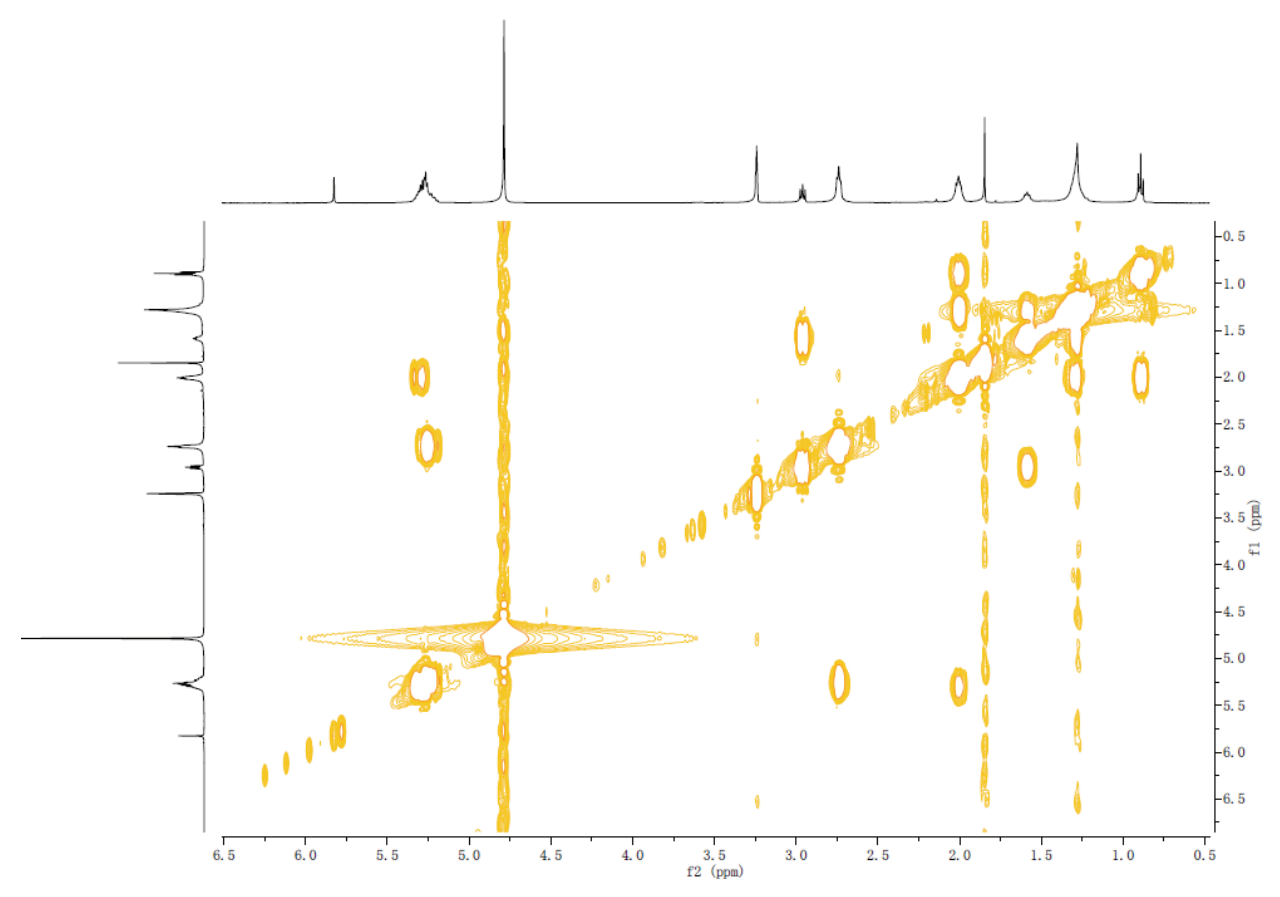

Figure S10. ${ }^{1} \mathrm{H}-{ }^{1} \mathrm{H}$ COSY spectrum of compound 2 


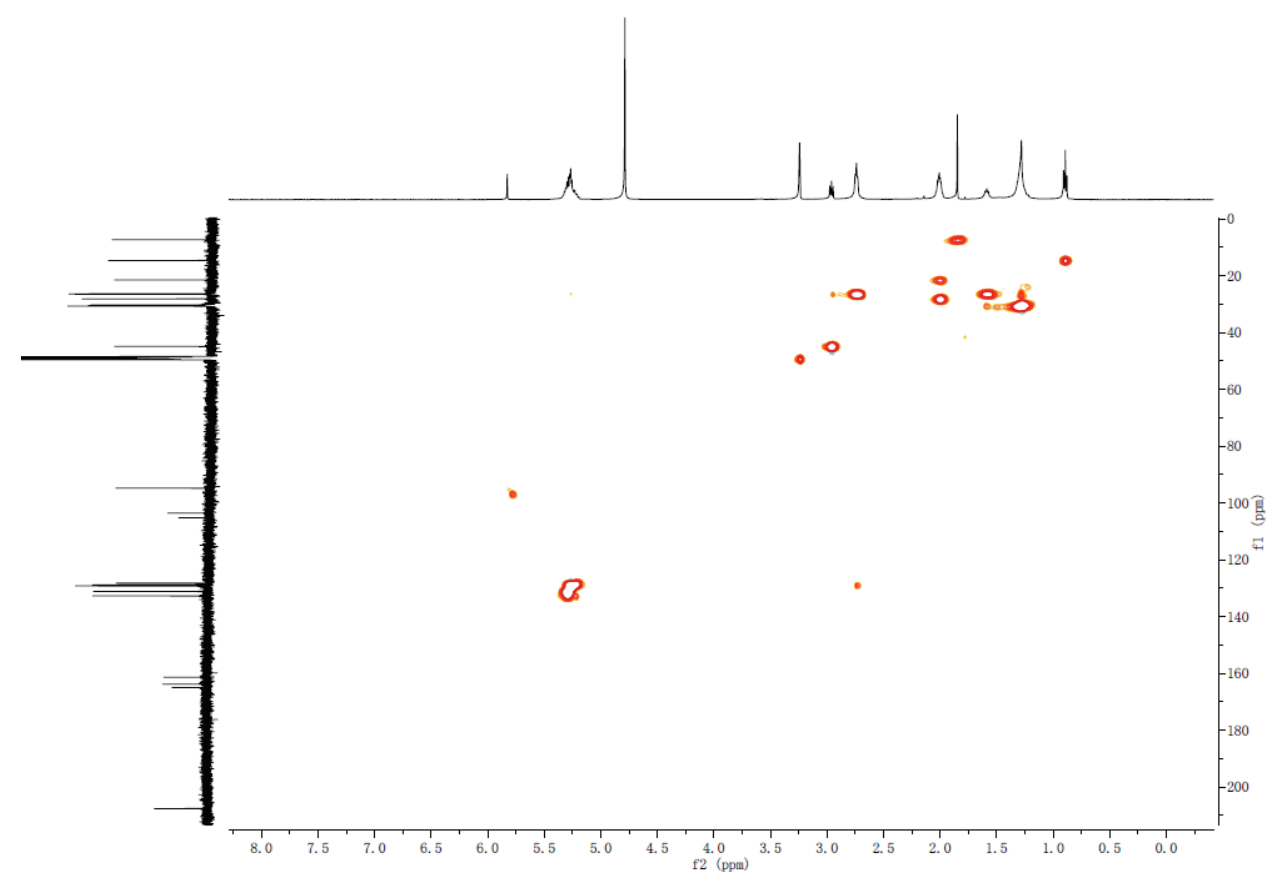

Figure S11. HSQC NMR spectrum of compound 2

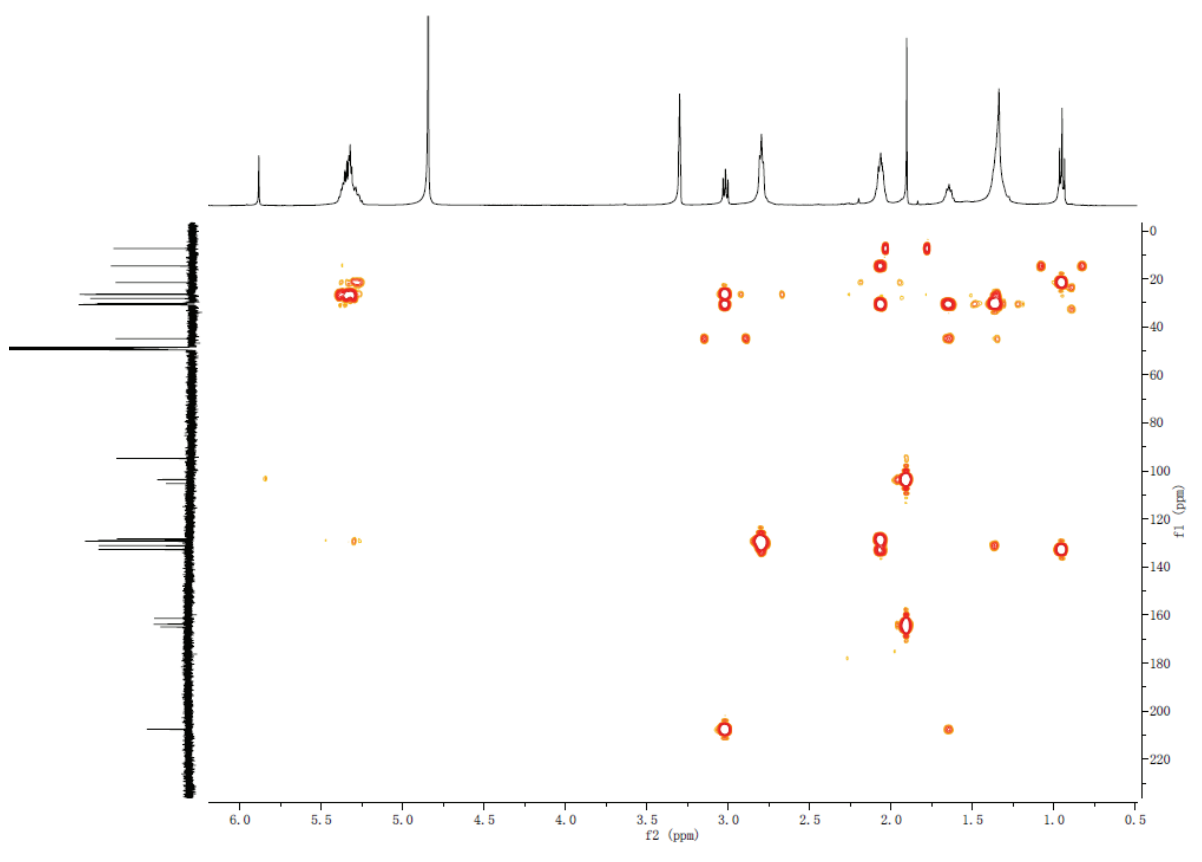

Figure S12. HMBC NMR spectrum of compound 2 


\begin{tabular}{|c|c|c|c|c|c|c|c|c|c|c|c|c|}
\hline 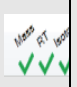 & Compound Name (Library Hit) & Formula & Intensity & Threshold & $\begin{array}{c}\text { Expected } \\
\mathrm{m} / \mathrm{z}\end{array}$ & $\begin{array}{c}\text { Found at } \\
\mathrm{m} / \mathrm{z}\end{array}$ & Error (ppm) & $\begin{array}{l}\text { Expected } \\
\text { RT (min) }\end{array}$ & $\begin{array}{c}\text { Found RT } \\
\text { (min) }\end{array}$ & $\begin{array}{l}\text { RT Delta } \\
\text { (min) }\end{array}$ & $\begin{array}{c}\text { Isotope Diff } \\
(\%)\end{array}$ & Purity (\%) \\
\hline$\checkmark \circ \checkmark$ & 388.26136 (No Match) & $\mathrm{C} 24 \mathrm{H} 36 \mathrm{O} 4$ & 8632892 & 50 & 389.2686 & 389.2688 & 0.5 & 0.00 & 0.08 & 0.08 & $0.8 \%$ & $0.0 \%$ \\
\hline
\end{tabular}
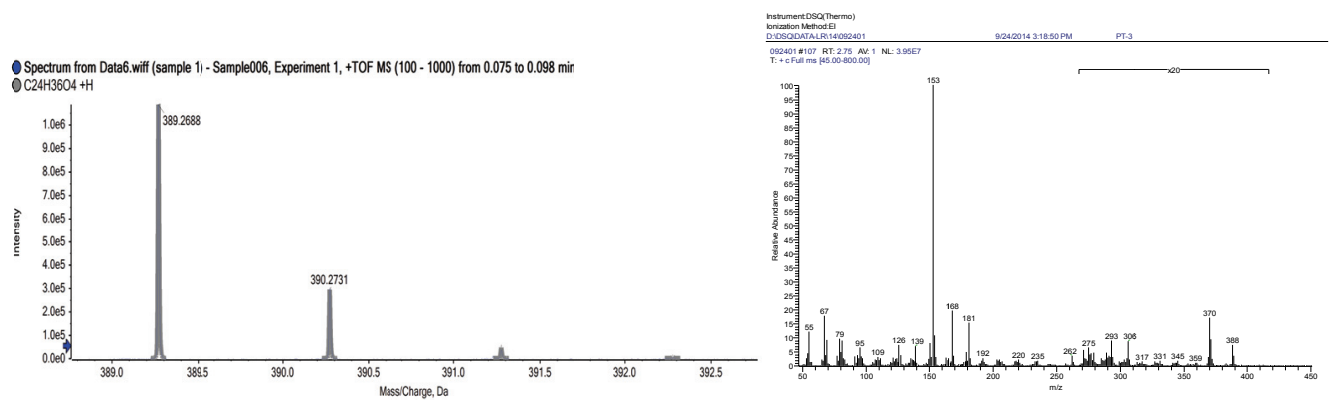

Figure S13. HRESIMS spectrum of compound 3

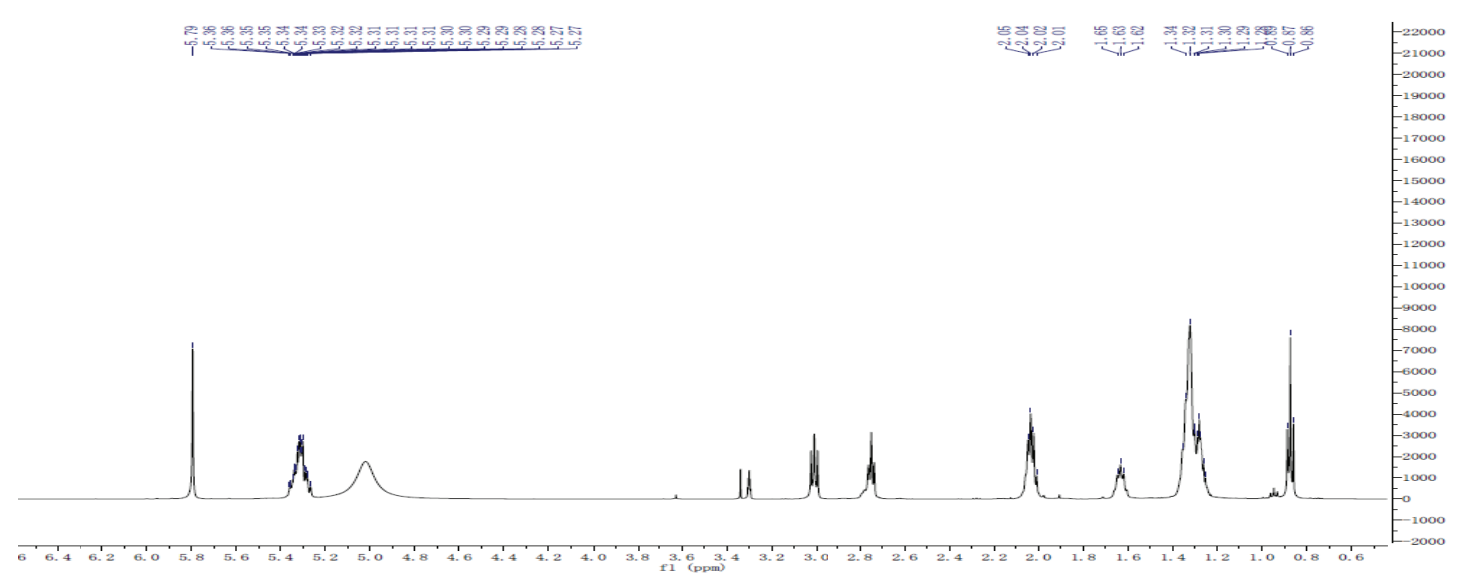

Figure S14. ${ }^{1} \mathrm{H}$ NMR (500 $\left.\mathrm{MHz}, \mathrm{CD}_{3} \mathrm{OD}\right)$ spectrum of compound 3 


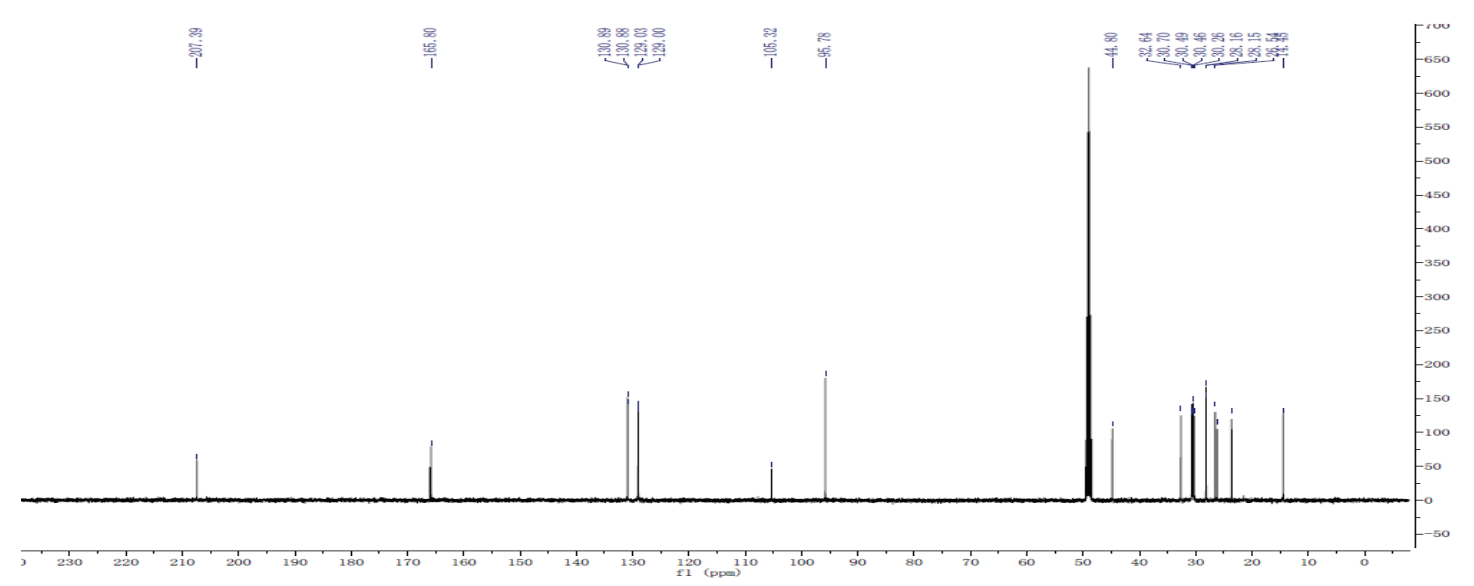

Figure S15. ${ }^{13} \mathrm{C}$ NMR (125 MHz, $\left.\mathrm{CD}_{3} \mathrm{OD}\right)$ spectrum of compound 3

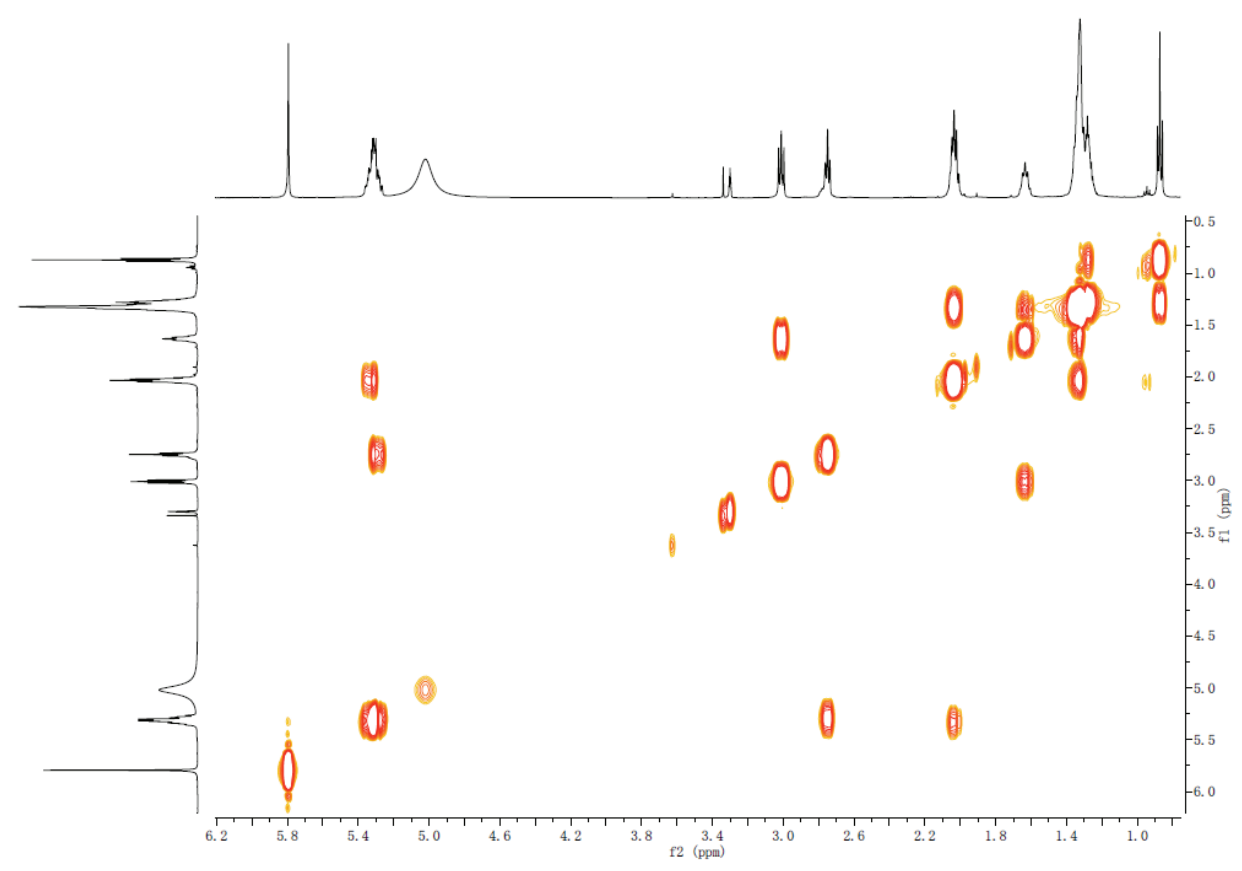

Figure S16. ${ }^{1} \mathrm{H}-{ }^{1} \mathrm{H}$ COSY spectrum of compound 3 


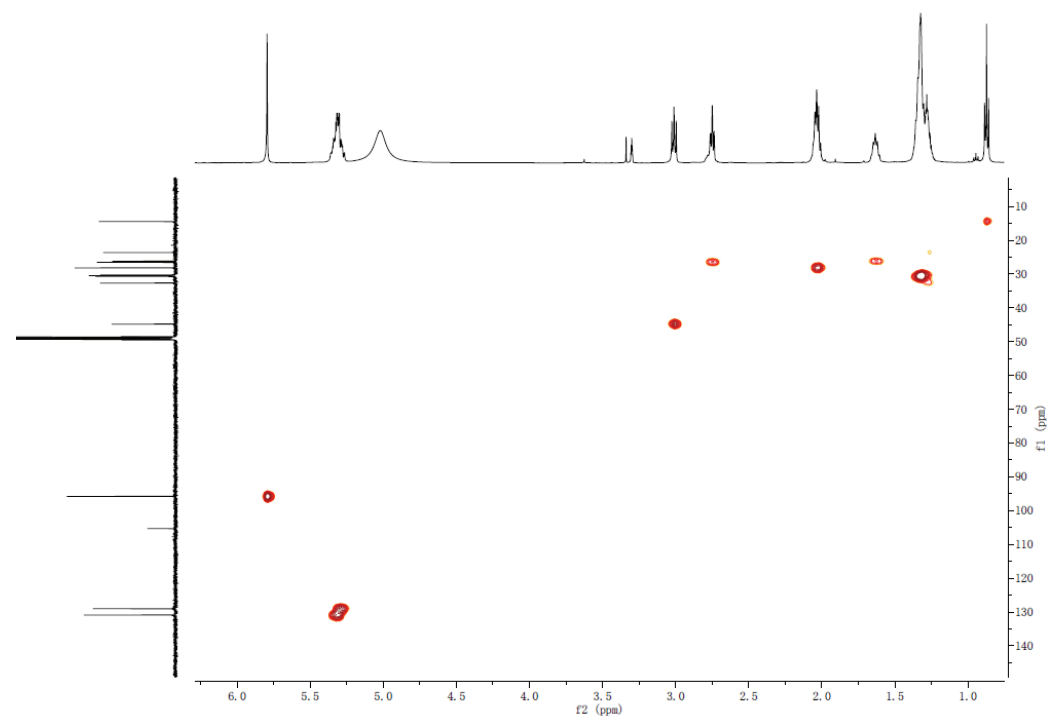

Figure S17. HSQC NMR spectrum of compound 3

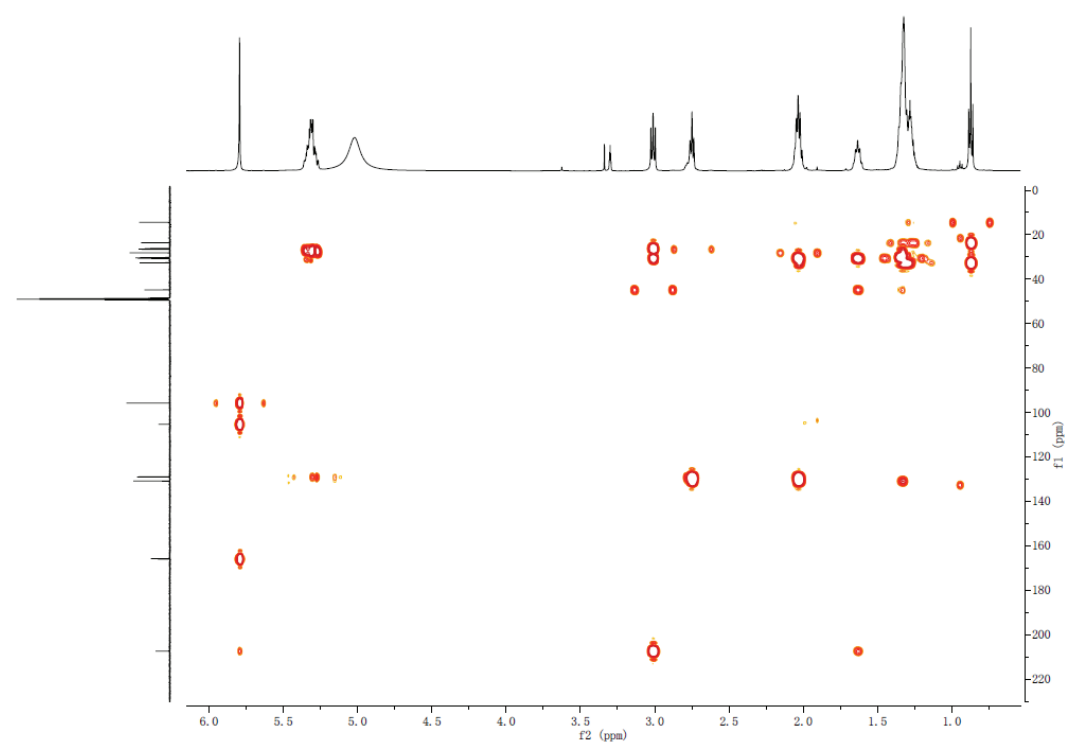

Figure S18. HMBC NMR spectrum of compound 3 


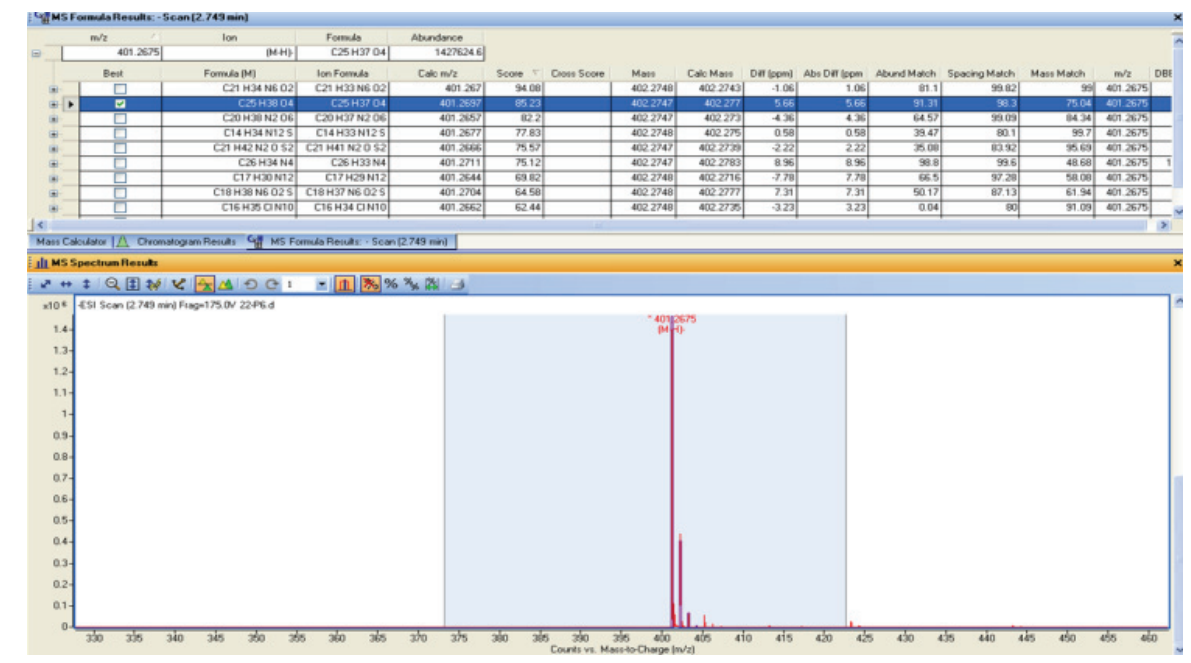

Figure S19. HRESIMS spectrum of compound 4

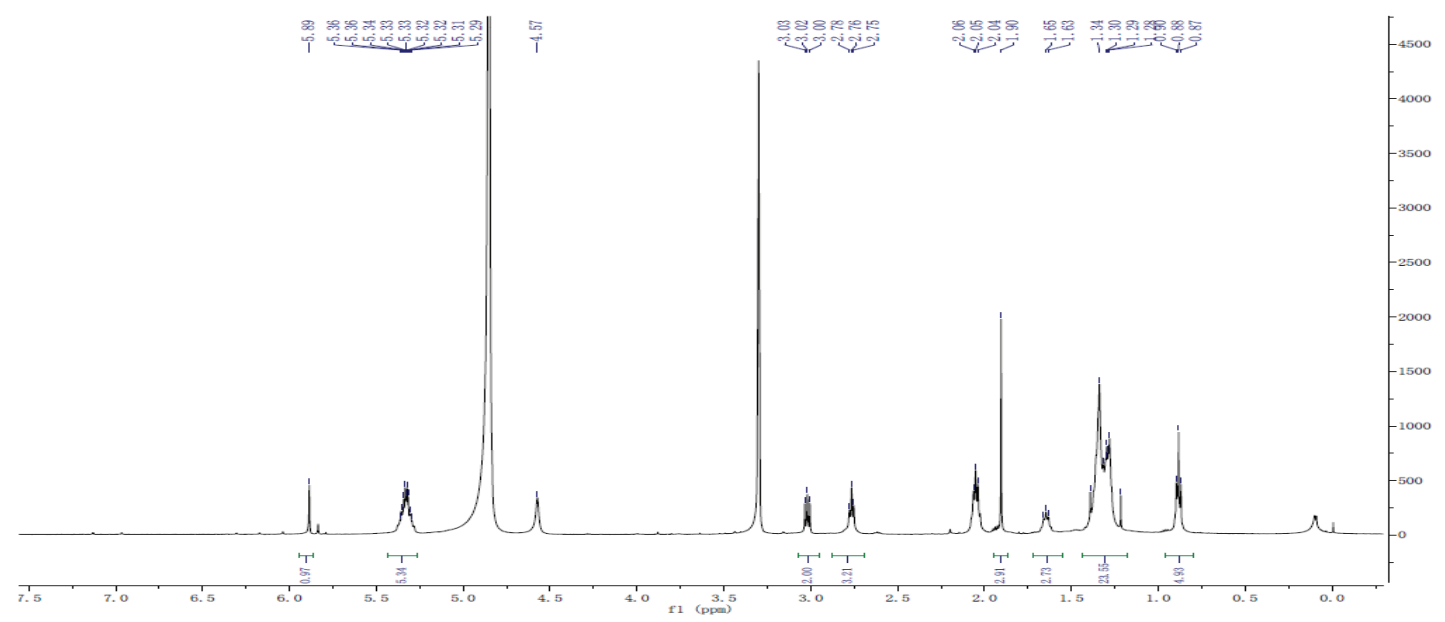

Figure S20. ${ }^{1} \mathrm{H}$ NMR (500 $\left.\mathrm{MHz}, \mathrm{CD}_{3} \mathrm{OD}\right)$ spectrum of compound 4 


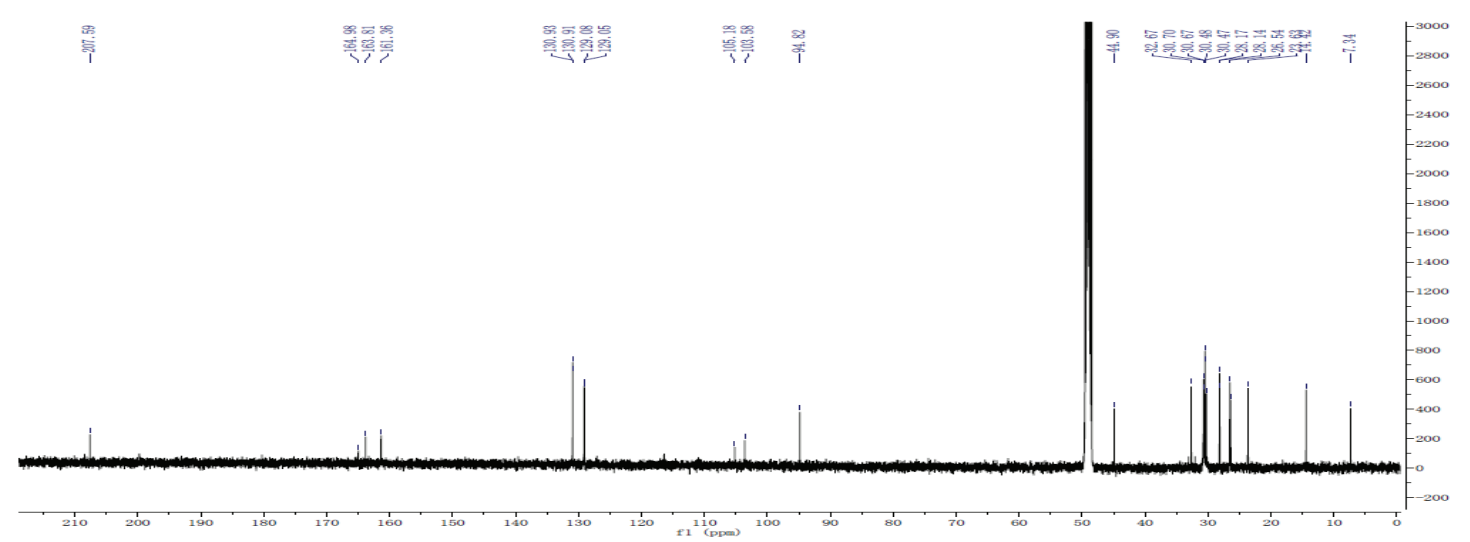

Figure S21. ${ }^{13} \mathrm{C}$ NMR (125 MHz, $\left.\mathrm{CD}_{3} \mathrm{OD}\right)$ spectrum of compound 4

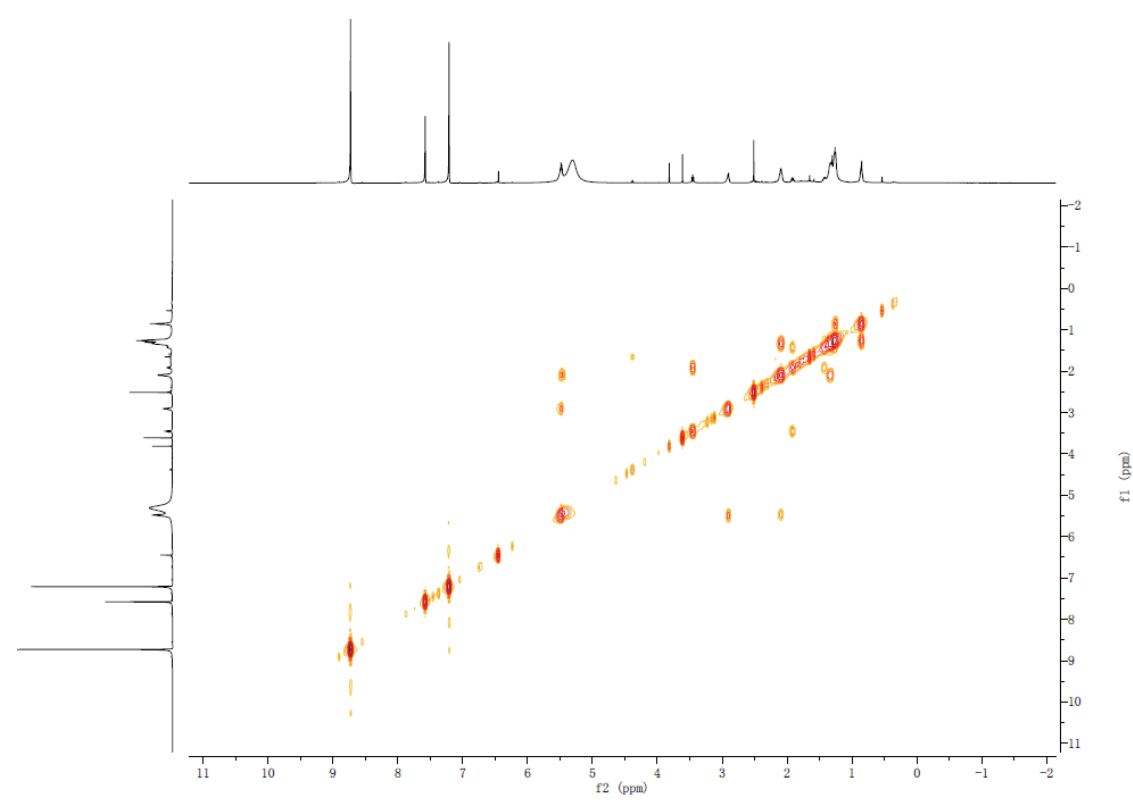

Figure S22. ${ }^{1} \mathrm{H}-{ }^{1} \mathrm{H}$ COSY spectrum of compound 4 


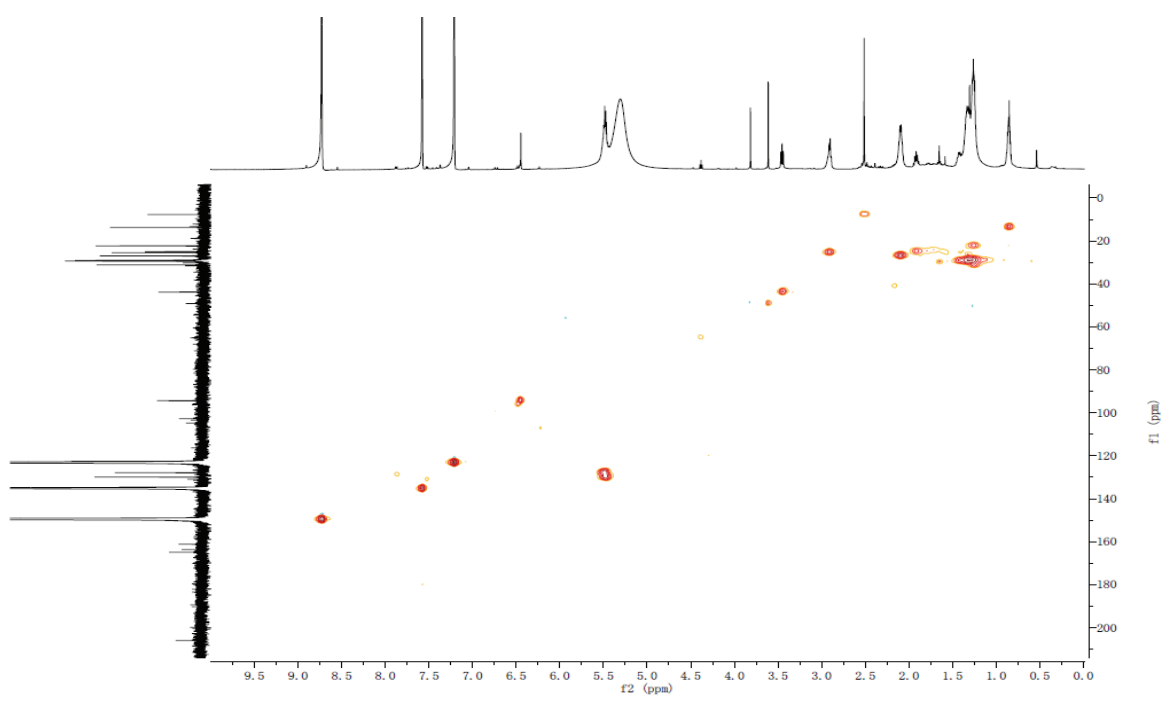

Figure S23. HSQC NMR spectrum of compound 4

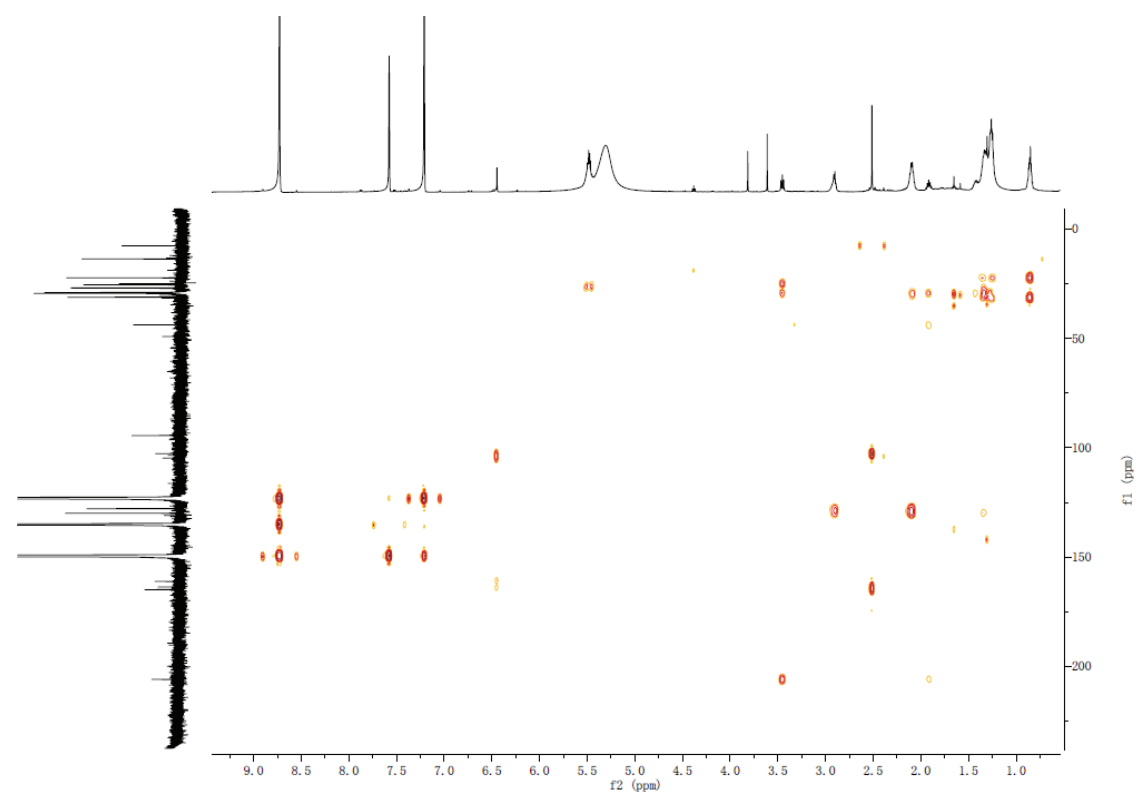

Figure S24. HMBC NMR spectrum of compound 4 


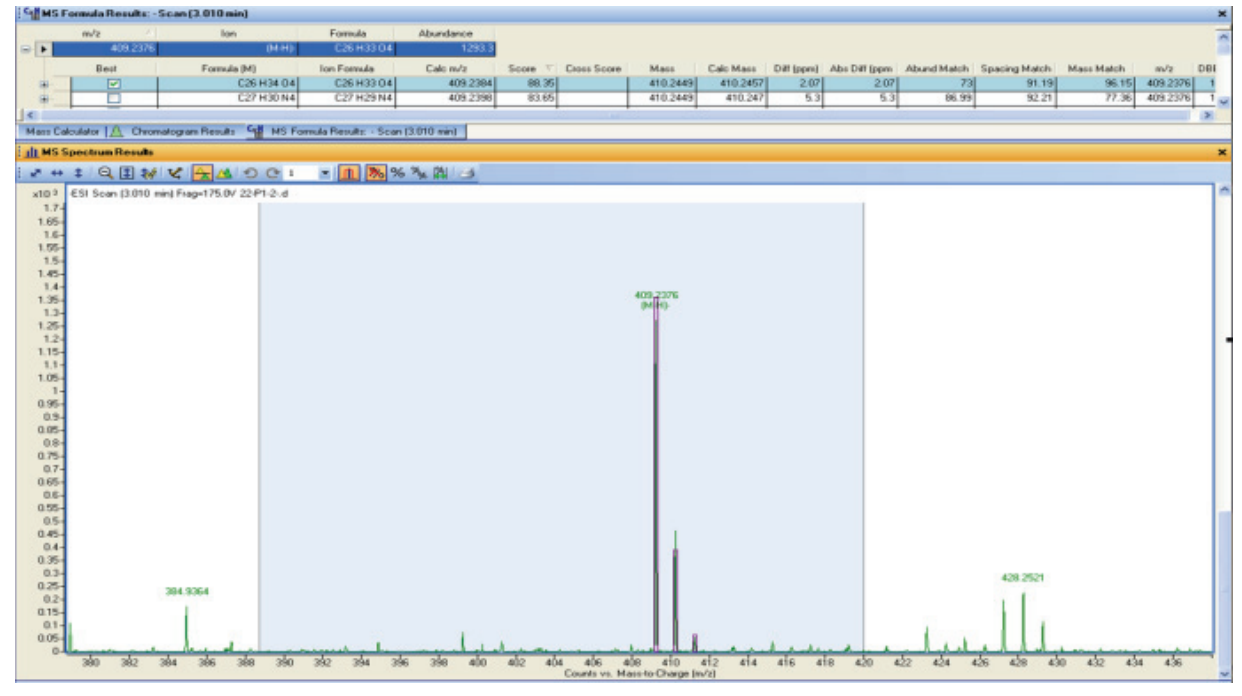

Figure S25. HRESIMS spectrum of compound 5

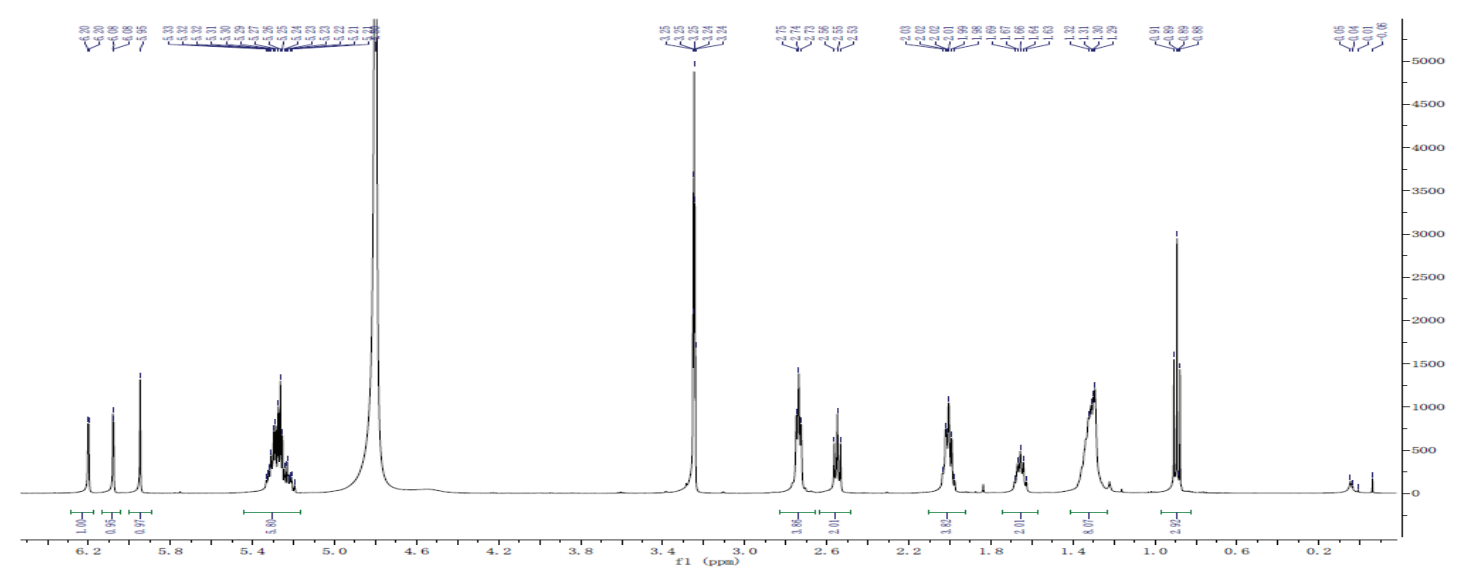

Figure S26. ${ }^{1} \mathrm{H}$ NMR ( $\left.500 \mathrm{MHz}, \mathrm{CD}_{3} \mathrm{OD}\right)$ spectrum of compound 5 


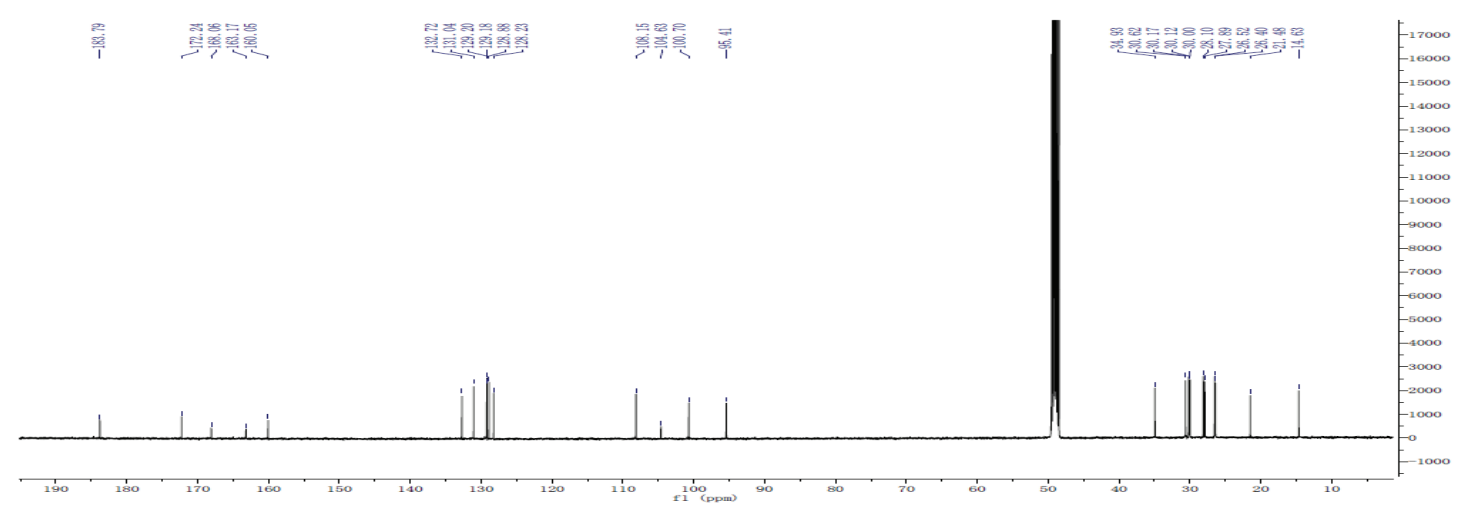

Figure S27. ${ }^{13} \mathrm{C}$ NMR (125 MHz, $\left.\mathrm{CD}_{3} \mathrm{OD}\right)$ spectrum of compound 5

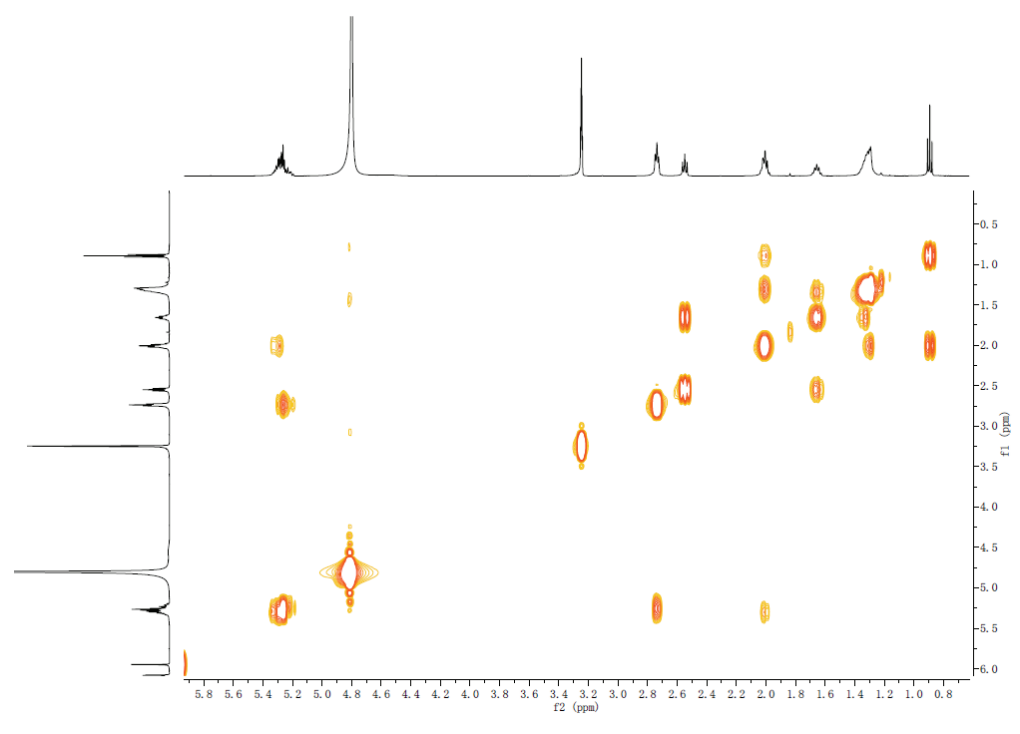

Figure S28. ${ }^{1} \mathrm{H}-{ }^{1} \mathrm{H}$ COSY spectrum of compound 5 


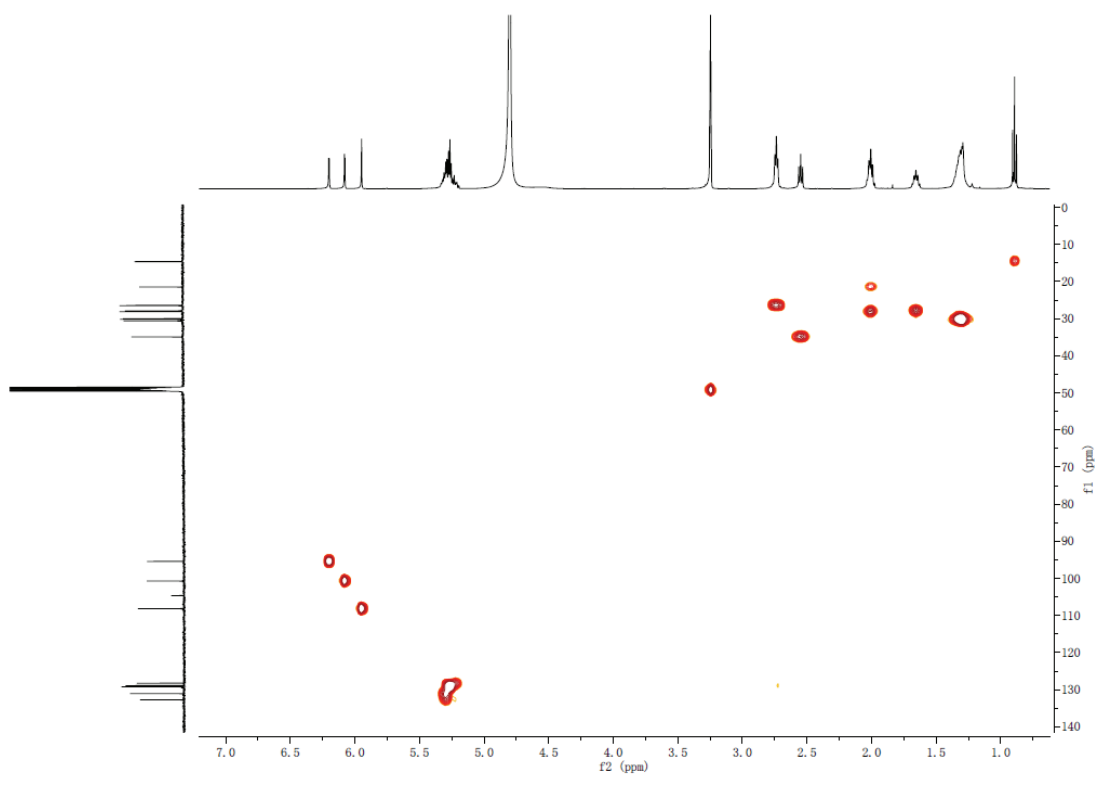

Figure S29. HSQC NMR spectrum of compound 5

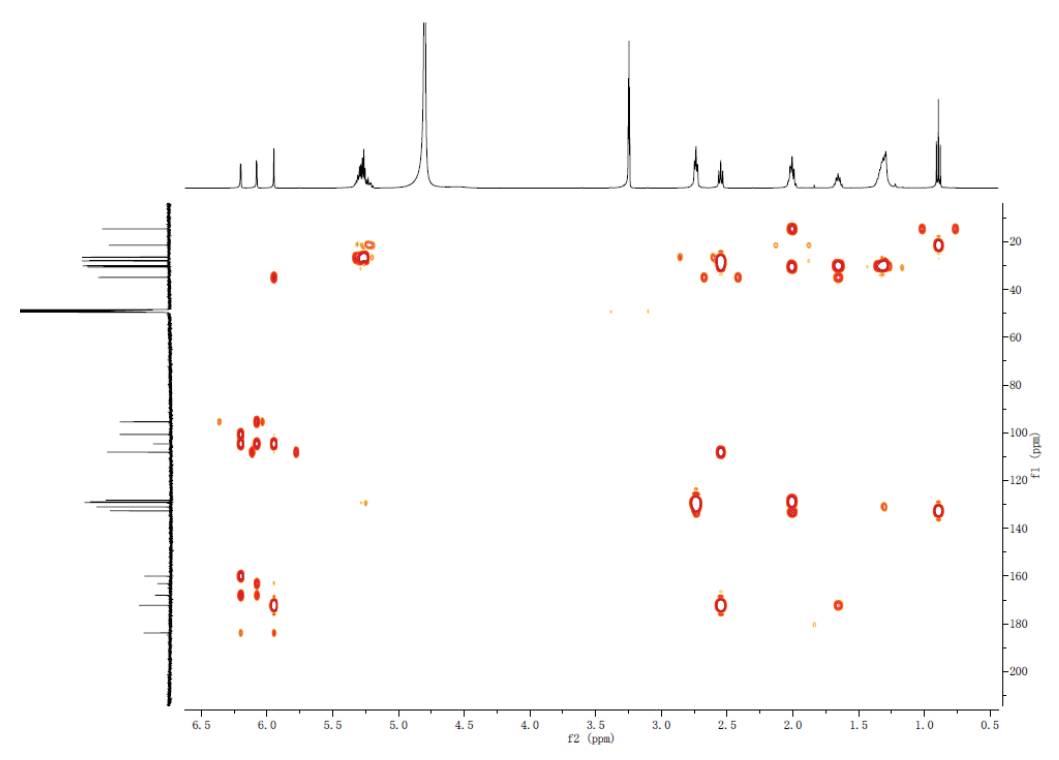

Figure S30. HMBC NMR spectrum of compound 5 


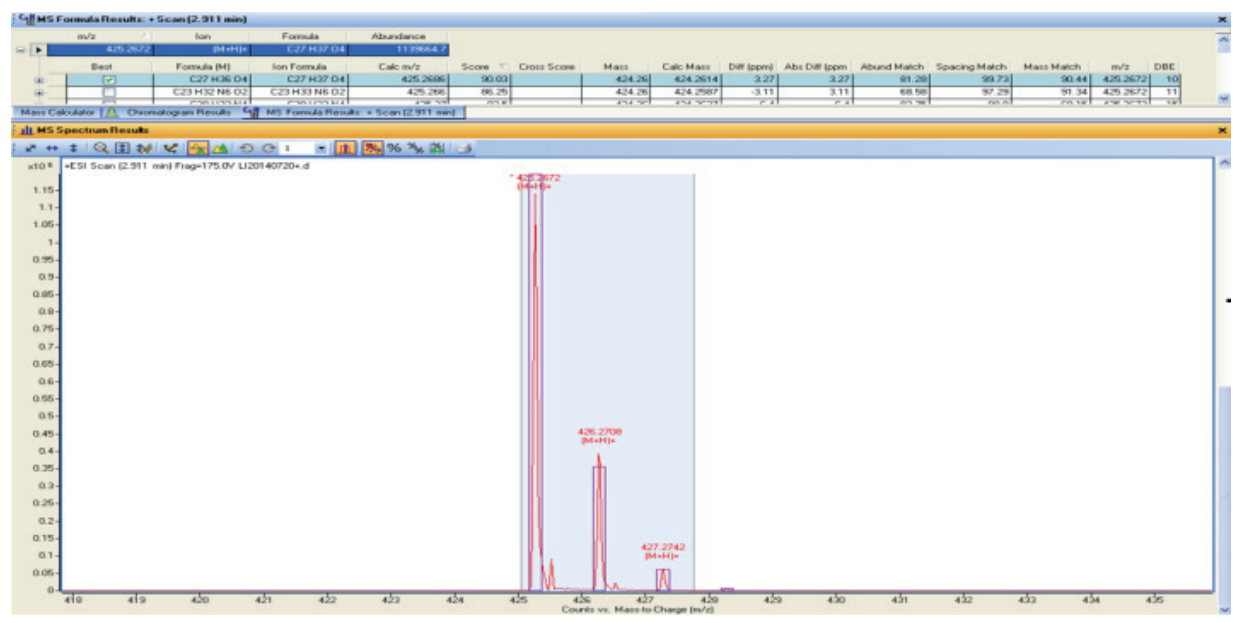

Figure S31. HRESIMS spectrum of compound 6

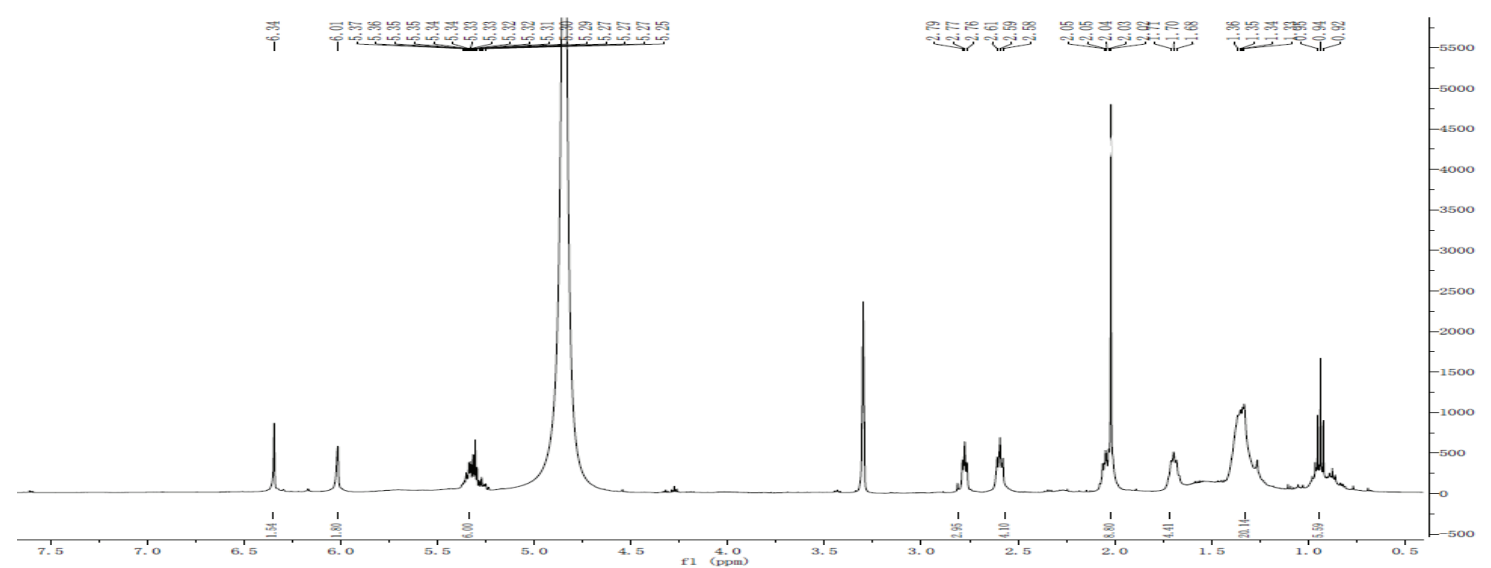

Figure S32. ${ }^{1} \mathrm{H}$ NMR ( $\left.500 \mathrm{MHz}, \mathrm{CD}_{3} \mathrm{OD}\right)$ spectrum of compound 6 


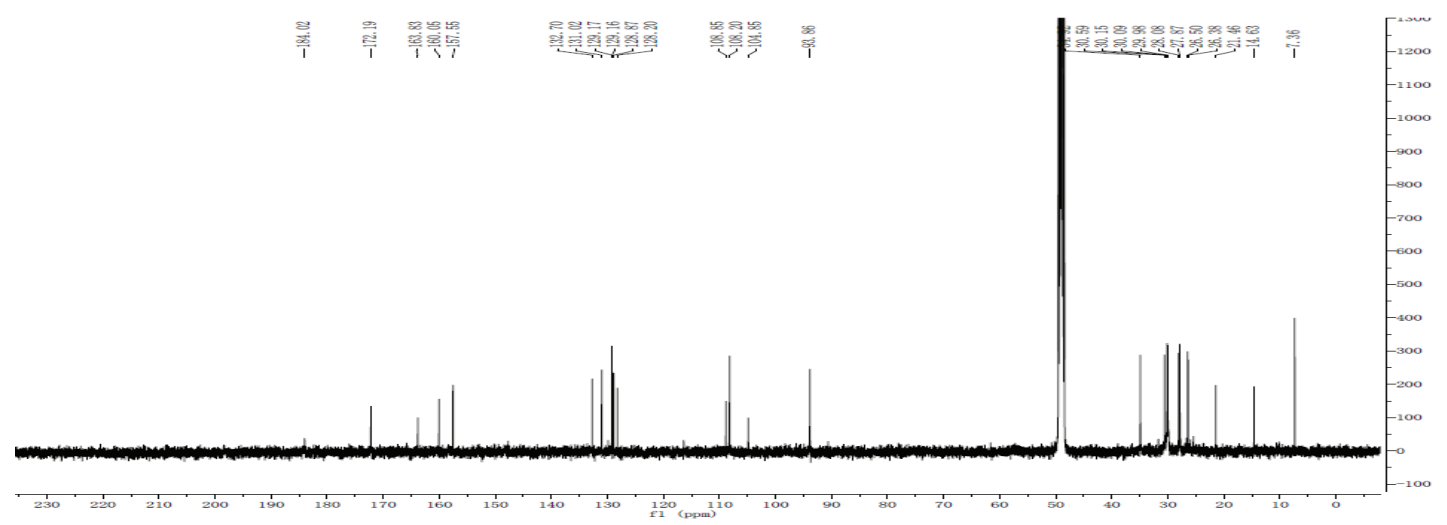

Figure S33. ${ }^{13} \mathrm{C}$ NMR (125 MHz, $\left.\mathrm{CD}_{3} \mathrm{OD}\right)$ spectrum of compound 6

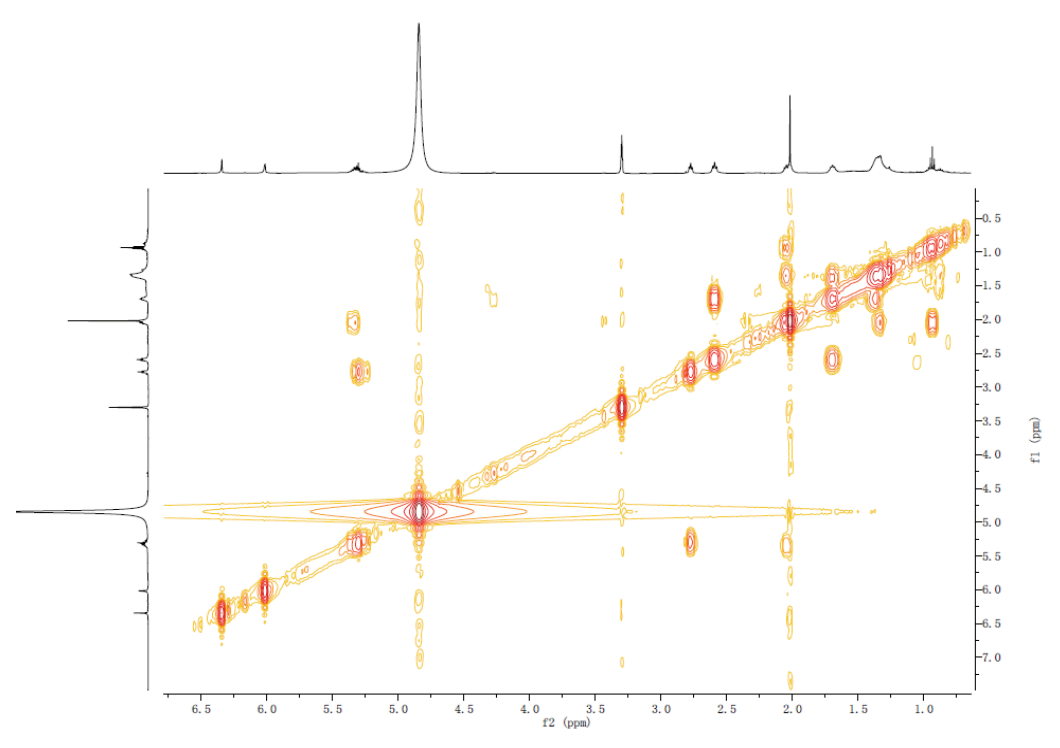

Figure S34. ${ }^{1} \mathrm{H}-{ }^{1} \mathrm{H}$ COSY spectrum of compound 6 


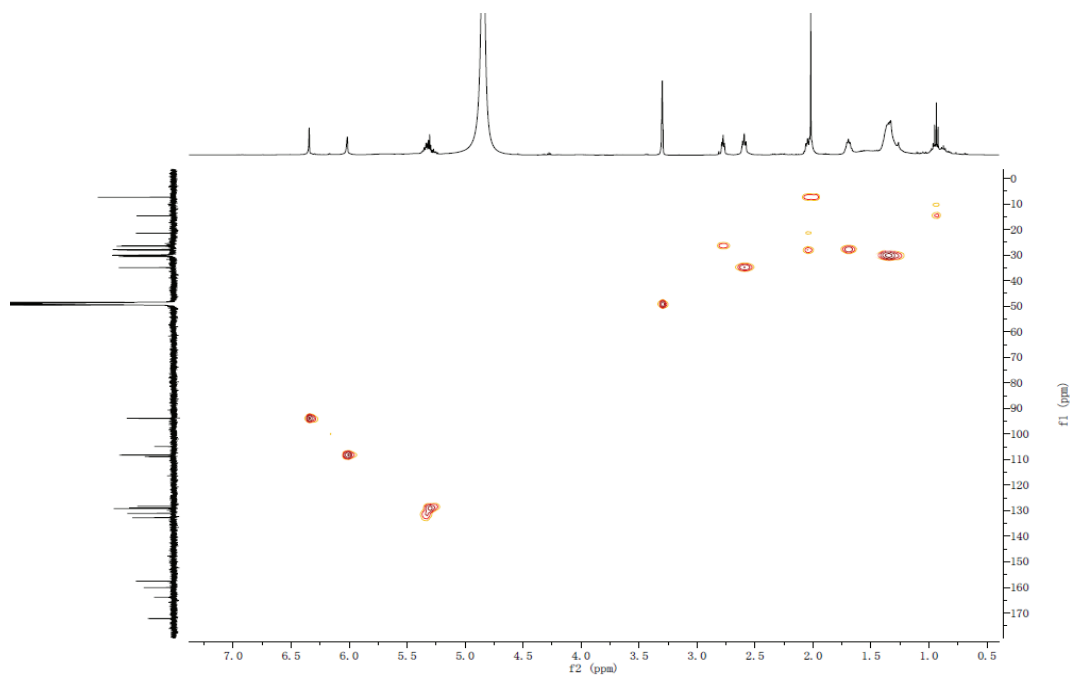

Figure S35. HSQC NMR spectrum of compound 6

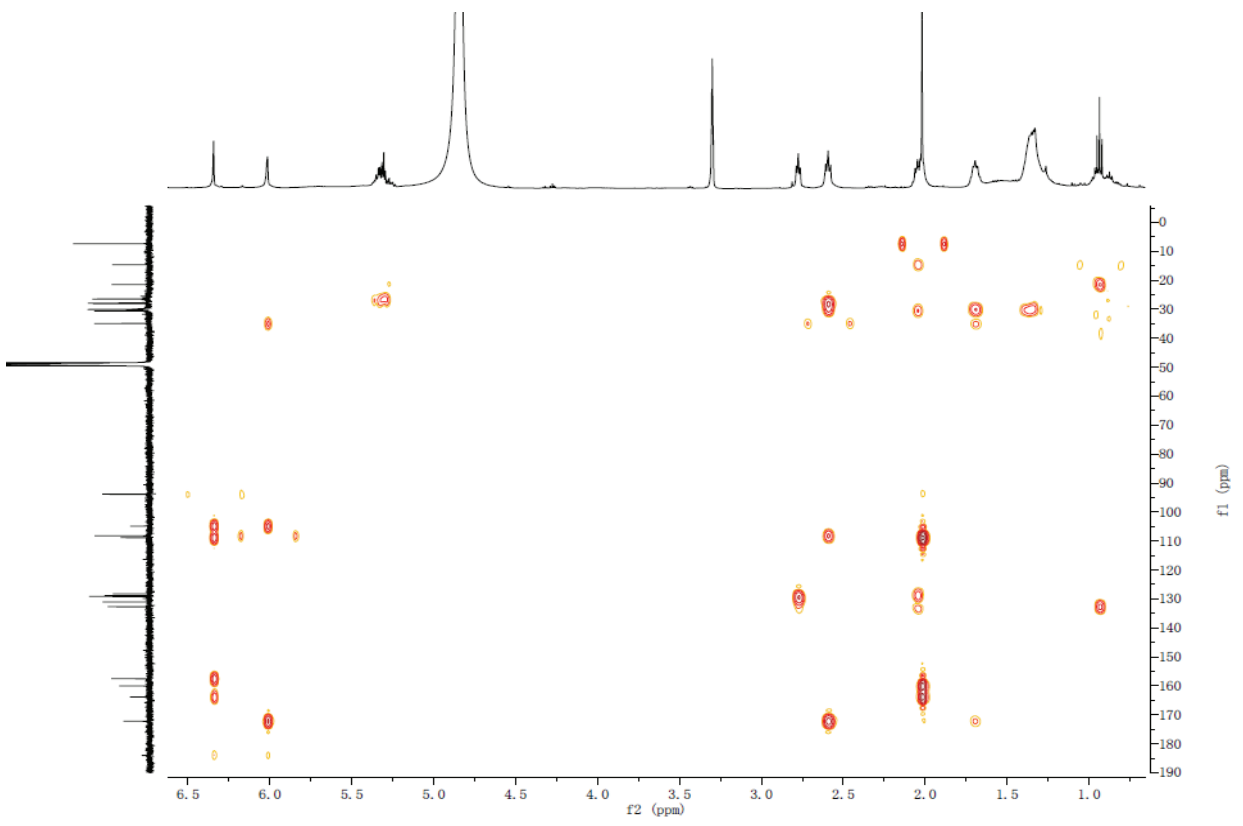

Figure S36. HMBC NMR spectrum of compound 6

18 


\begin{tabular}{|c|c|c|c|c|c|c|c|c|c|c|c|c|}
\hline$v$ & Compound Name (Library Hit) & Formula & Intensity & Threshold & $\begin{array}{c}\text { Expected } \\
\mathrm{m} / \mathrm{z}\end{array}$ & $\begin{array}{c}\text { Found at } \\
\mathrm{m} / \mathbf{z}\end{array}$ & $\begin{array}{l}\text { Error } \\
\text { (ppm) }\end{array}$ & $\begin{array}{l}\text { Expected } \\
\text { RT (min) }\end{array}$ & $\begin{array}{c}\text { Found RT } \\
\text { (min) }\end{array}$ & $\begin{array}{l}\text { RT Delta } \\
\text { (min) }\end{array}$ & $\begin{array}{l}\text { Isotope } \\
\text { Diff (\%) }\end{array}$ & Purity (\%) \\
\hline$\checkmark 0$ & 412.26136 (No Match) & $\mathrm{C} 26 \mathrm{H} 36 \mathrm{O} 4$ & 9591703 & 50 & 413.2686 & 413.2683 & -0.8 & 0.00 & 0.13 & 0.13 & $10.2 \%$ & $0.0 \%$ \\
\hline
\end{tabular}

O Spectrum from Data1.wiff (sample 1) - Sample001, Experiment 1, +TOF MS (100 - 1000) from 0.179 to $0.203 \mathrm{~min}$ $\mathrm{O} \mathrm{C} 26 \mathrm{H} 36 \mathrm{O} 4+\mathrm{H}$

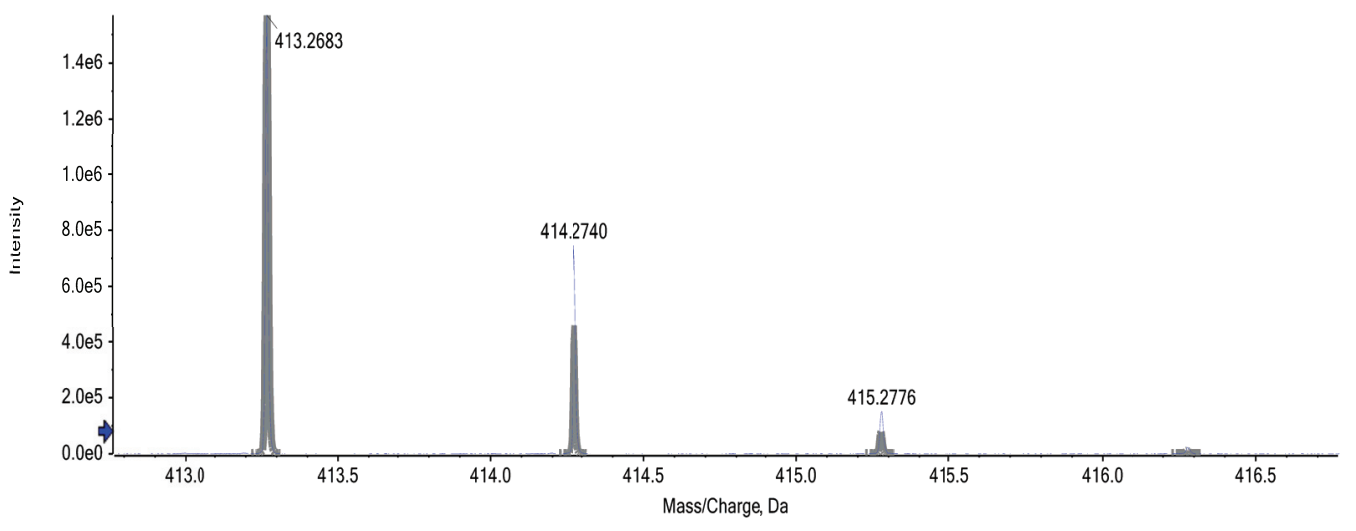

Figure S37. HRESIMS spectrum of compound 7

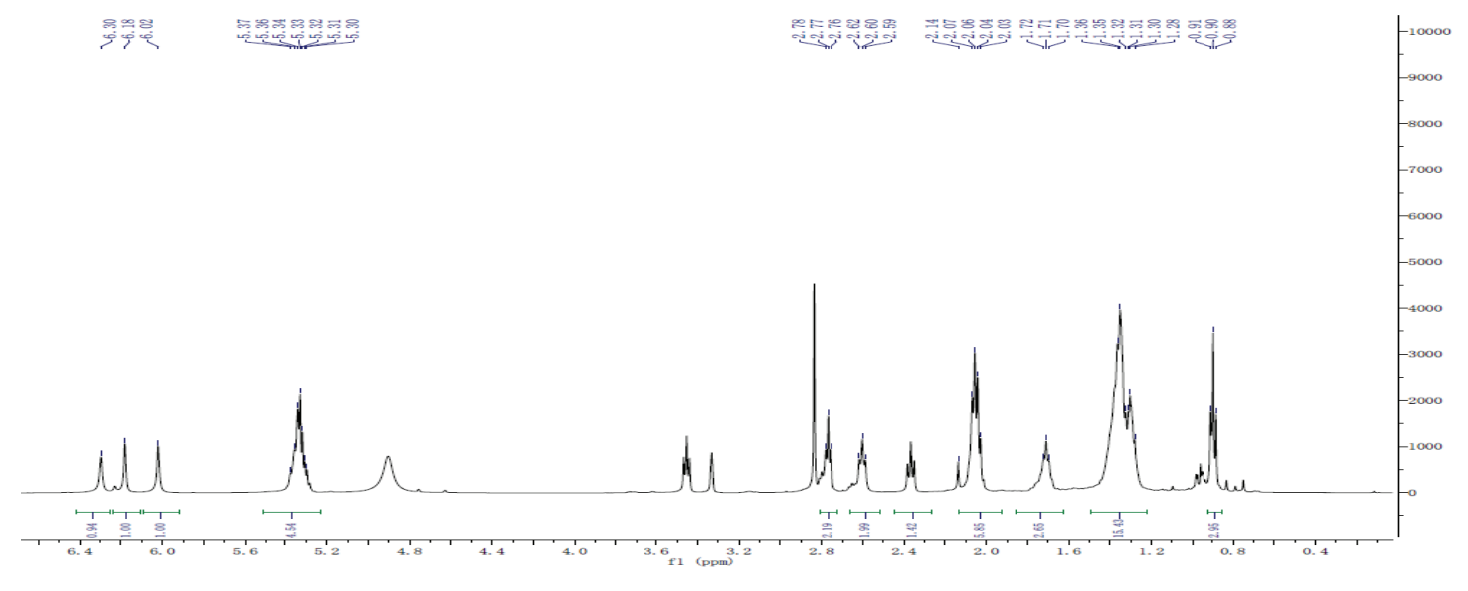

Figure S38. ${ }^{1} \mathrm{H}$ NMR (500 $\left.\mathrm{MHz}, \mathrm{CD}_{3} \mathrm{OD}\right)$ spectrum of compound 7 


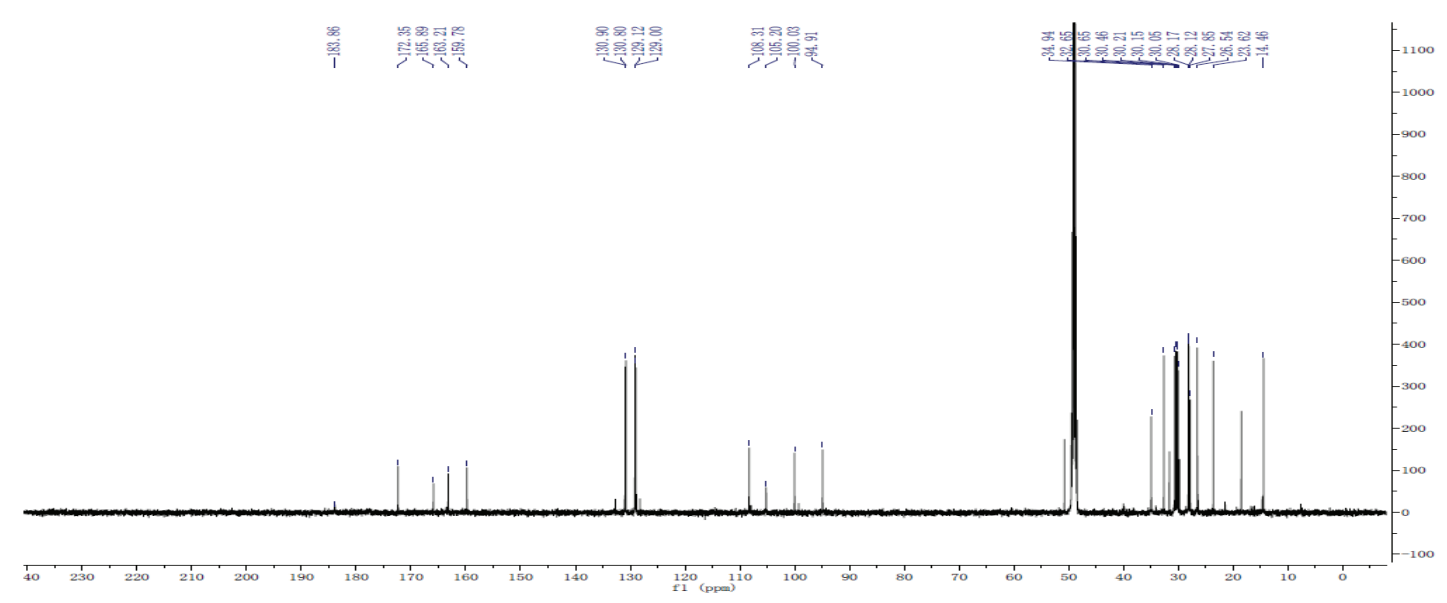

Figure S39. ${ }^{13} \mathrm{C}$ NMR (125 MHz, $\left.\mathrm{CD}_{3} \mathrm{OD}\right)$ spectrum of compound 7

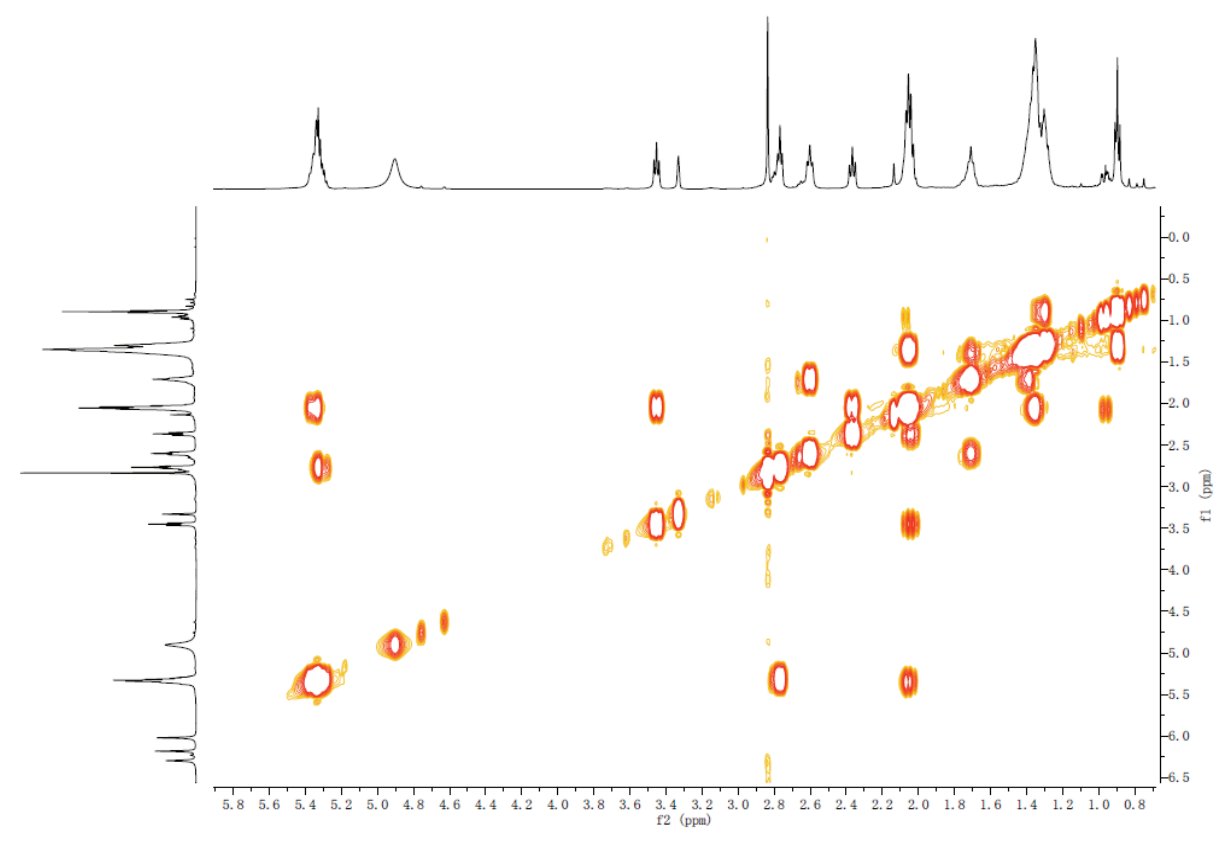

Figure S40. ${ }^{1} \mathrm{H}-{ }^{1} \mathrm{H}$ COSY spectrum of compound 7 


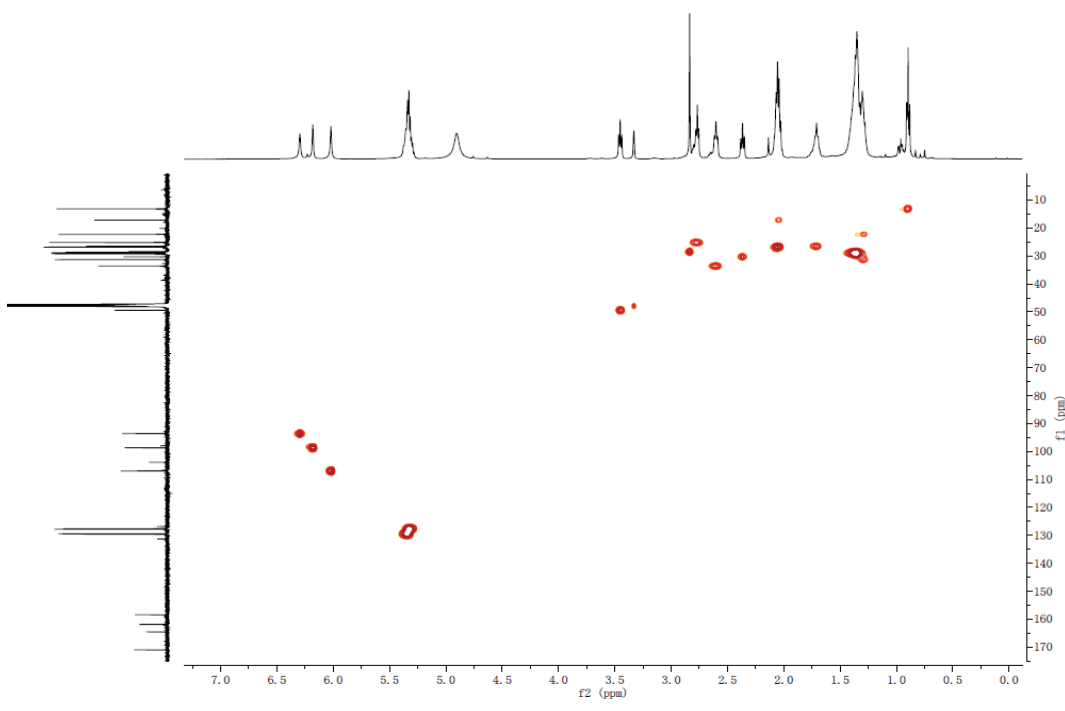

Figure S41. HSQC NMR spectrum of compound 7

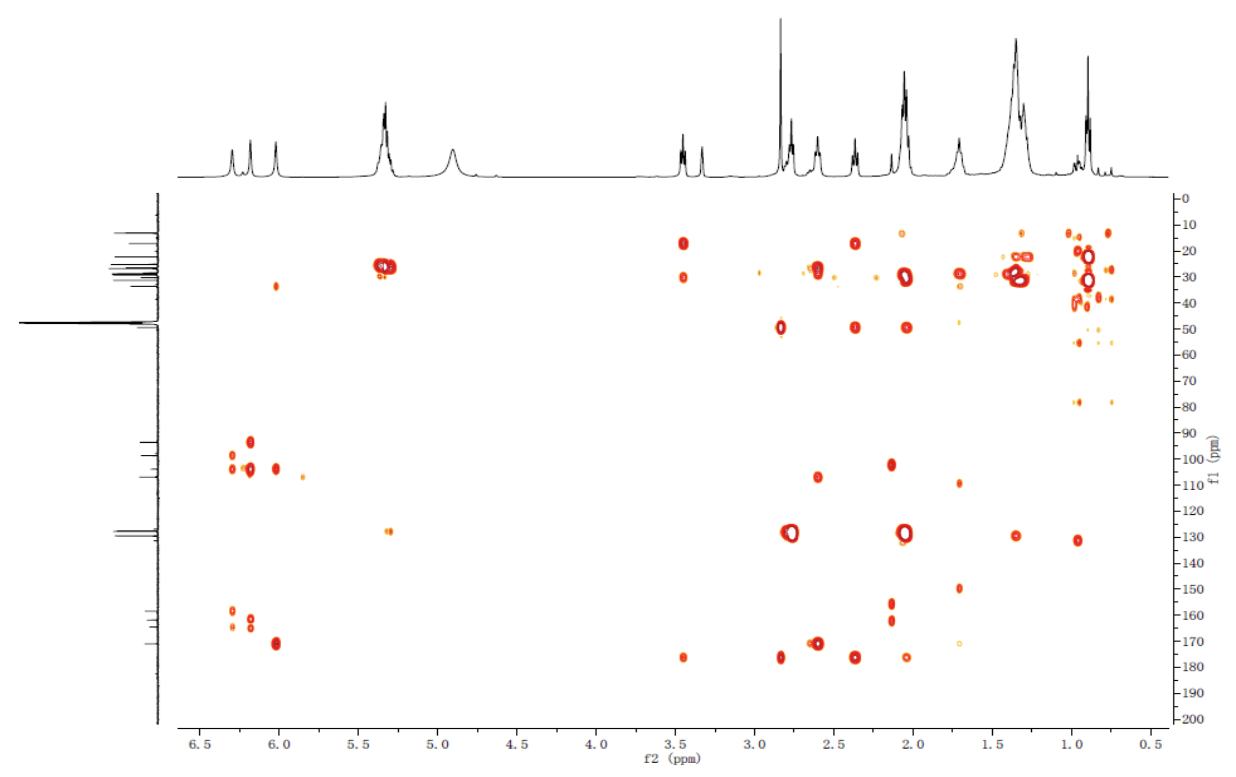

Figure S42. HMBC NMR spectrum of compound 7 